\title{
INTEGRAL CONTROL OF INFINITE-DIMENSIONAL SYSTEMS IN THE PRESENCE OF HYSTERESIS: AN INPUT-OUTPUT APPROACH*
}

\author{
Hartmut Logemann $^{1}$, Eugene P. Ryan ${ }^{1}$ and Ilya Shvartsman ${ }^{1}$
}

\begin{abstract}
This paper is concerned with integral control of systems with hysteresis. Using an inputoutput approach, it is shown that application of integral control to the series interconnection of either (a) a hysteretic input nonlinearity, an $L^{2}$-stable, time-invariant linear system and a non-decreasing globally Lipschitz static output nonlinearity, or (b) an $L^{2}$-stable, time-invariant linear system and a hysteretic output nonlinearity, guarantees, under certain assumptions, tracking of constant reference signals, provided the positive integrator gain is smaller than a certain constant determined by a positivity condition in the frequency domain. The input-output results are applied in a general state-space setting wherein the linear component of the interconnection is a well-posed infinite-dimensional system.
\end{abstract}

Mathematics Subject Classification. 34G20, 47J40, 47N70, 93C23, 93C25, 93D10, 93D25.

Received September 27, 2005. Revised March 20, 2006.

Published online June 5, 2007.

\section{INTRODUCTION}

This paper is concerned with integral control of systems with input or output hysteresis. Consider the system shown in Figure 1, where $u$ is the input, $\Phi$ is a hysteresis nonlinearity, $G$ is the input-output operator of an $L^{2}$-stable time-invariant linear system, the signal $g \in L^{2}\left(\mathbb{R}_{+}\right)$models the effect of initial conditions, $\psi$ is a non-decreasing globally Lipschitz static nonlinearity and $y$ is the output. The operator $\Phi$ belongs to a class of hysteresis operators with certain natural monotonicity and Lipschitz continuity properties and which contains, in particular, operators of backlash, elastic-plastic and, more generally, of Prandtl and Preisach type (see Sect. 3). In Section 4, we show that applying integral control to the system in Figure 1 guarantees tracking of constant reference signals, in the presence of output disturbances, provided that a number of natural assumptions hold. In particular, it is assumed that (a) the steady-state gain of the linear part of the plant is positive, (b) the positive time-dependent integrator gain is ultimately smaller than some constant determined by a positivity condition in the frequency domain, (c) the output disturbance is of a particular class which encompasses sums of constant signals and weighted $L^{2}$-signals and (d) the reference value is feasible in a natural sense to be made precise in due course.

\footnotetext{
Keywords and phrases. Actuator nonlinearities, hysteresis, infinite-dimensional systems, input-output analysis, integral control, sensor nonlinearities.

* This work was supported by UK EPSRC Grant GR/S94582/01.

1 Department of Mathematical Sciences, University of Bath, Bath BA2 7AY, UK; hl@maths.bath.ac.uk; epr@maths.bath.ac.uk; shvartia@muohio.edu

(C) EDP Sciences, SMAI 2007
} 


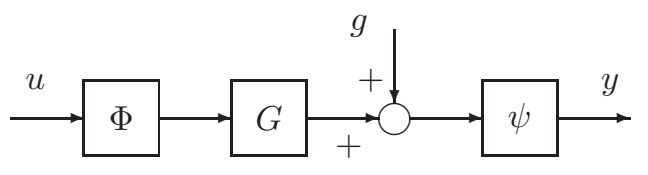

FIGURE 1. System with hysteretic input and static output nonlinearity.

In Section 5, we deal with integral control of linear systems with output hysteresis. Consider the system shown in Figure 2, where the hysteresis operator $\Phi$ is in the plant output.

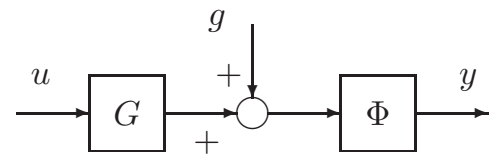

FiguRE 2. System with output hysteresis.

Similar to the case of input hysteretic nonlinearities, we show that applying integral control to the system in Figure 2 guarantees tracking of constant reference signals (again assumed feasible in a natural sense), in the presence of output disturbances, under somewhat different assumptions. In particular, it is assumed that (a) $G$ is a convolution operator with kernel in $L^{2}\left(\mathbb{R}_{+}\right)+\mathbb{R} \delta_{0}\left(\delta_{0}\right.$ is the unit point mass at $\left.t=0\right)$ and positive steady-state gain, (b) the positive constant integrator gain is smaller than some number determined by a positivity condition in the frequency domain, (c) the disturbance is the sum of a constant signal and a $L^{2}$-signal with zero limit at infinity. In Section 6, the input-output results of Sections 4 and 5 are applied in a general state-space setting wherein the linear component of the interconnection belongs to the class of well-posed infinite-dimensional systems, see $[23-25,29]$. We remark that this class is rather general; it includes many distributed parameter systems and all retarded and neutral time-delay systems which are of interest in control engineering applications (see Rem. 6.1 for more details).

Some preliminaries are presented in Section 2 and proofs of existence and uniqueness of solutions to the functional differential equations arising in Sections 4 and 5 are provided in the Appendix.

Systems of the form shown in Figures 1 and 2, where $\Phi$ is a hysteretic nonlinearity are common in control engineering. In particular, hysteresis plays an important role in the context of smart material actuators and sensors, see, for example, $[1,7,26]$. There has recently been considerable effort in the development of rigorous results on stability and control of systems with hysteresis, see, for example $[7,11-13,16,17,19,26,27]$. This paper represents a further contribution to this effort. We remark that the results in this paper are new even in the case where $G$ is the input-output operator of a finite-dimensional system. Finally, we mention that in [16], a state-space approach to low-gain integral control of exponentially stable regular infinite-dimensional systems with input hysteresis (in the absence of output nonlinearity) was developed. We emphasize that the inputoutput approach presented in this paper not only complements but, more importantly, substantially extends and improves the results in [16].

Notation and terminology. Let $\mathbb{C}_{+}$denote the open right half of the complex plane. The space of all complex-valued, bounded and holomorphic functions defined on $\mathbb{C}_{+}$is denoted by $H^{\infty}\left(\mathbb{C}_{+}\right)$. Let $I$ be an interval of the form $I=[0, T)$, where $0<T \leq \infty$. The space of real-valued (locally) absolutely continuous functions defined on an interval $I$ is denoted by $W_{\mathrm{loc}}^{1,1}(I)$ : that is, a function $f: I \rightarrow \mathbb{R}$ is in $W_{\mathrm{loc}}^{1,1}(I)$ if and only if there exists a locally integrable function $g: I \rightarrow \mathbb{R}$ such that

$$
f(t)=f(0)+\int_{0}^{t} g(\tau) \mathrm{d} \tau, \quad \forall t \in[0, T) .
$$


Write $\mathbb{R}_{+}:=[0, \infty)$. Let $\theta: \mathbb{R}_{+} \rightarrow \mathbb{R}$ denote the function which is identically equal to 1 , that is,

$$
\theta(t)=1, \quad \forall t \in \mathbb{R}_{+} .
$$

The unit point mass at $t=\tau$ (Dirac distribution with support at $t=\tau$ ) is denoted by $\delta_{\tau}$. As usual, $\mathcal{B}(X, Y)$ denotes the space of bounded linear operators from a normed vector space $X$ into a normed vector space $Y$ : if $X=Y$, then we simply write $\mathcal{B}(X)$.

Let $0<T \leq \infty$ and $\tau \in(0, T)$. We define $P_{\tau}: L_{\text {loc }}^{p}([0, T)) \rightarrow L^{p}\left(\mathbb{R}_{+}\right)$by

$$
\left(P_{\tau} u\right)(t)= \begin{cases}u(t), & 0 \leq t \leq \tau, \\ 0, & t>\tau .\end{cases}
$$

Furthermore, let $I=[0, T]$, where $0<T<\infty$, or $I=[0, T)$, where $0<T \leq \infty$. For $\tau \in I$ we define $Q_{\tau}: C(I) \rightarrow C\left(\mathbb{R}_{+}\right)$

$$
\left(Q_{\tau} u\right)(t)= \begin{cases}u(t), & 0 \leq t \leq \tau, \\ u(\tau), & t>\tau .\end{cases}
$$

Let $X$ and $Y$ be subsets of any of the spaces $C\left(\mathbb{R}_{+}\right)$or $L_{\text {loc }}^{p}\left(\mathbb{R}_{+}\right)$, where $1 \leq p \leq \infty$. An operator $\Psi: X \rightarrow Y$ is said to be causal if, for all $\tau \geq 0$ and all $v_{1}, v_{2} \in X, v_{1}=v_{2}$ on $[0, \tau]$ implies that $\Psi\left(v_{1}\right)=\Psi\left(v_{2}\right)$ on $[0, \tau]$. Causality can be expressed using the operators $P_{\tau}$ and $Q_{\tau}$ : if $X \subset L_{\mathrm{loc}}^{p}\left(\mathbb{R}_{+}\right)$and $P_{\tau} X \subset X$ for all $\tau \geq 0$, then $\Psi$ is causal if and only if

$$
P_{\tau} \Psi\left(P_{\tau} v\right)=P_{\tau} \Psi(v), \quad \forall \tau>0, \quad \forall v \in X
$$

if $X \subset C\left(\mathbb{R}_{+}\right)$and $Q_{\tau} X \subset X$ for all $\tau \geq 0$, then $\Psi$ is causal if and only if

$$
P_{\tau} \Psi\left(Q_{\tau} v\right)=P_{\tau} \Psi(v), \quad \forall \tau \geq 0, \quad \forall v \in X
$$

The following simple, but important, remark shows that causal operators can be extended to the "localized" version of the domain space and to spaces of functions with a finite time horizon.

Remark 1.1. Let $0<T \leq \infty$ and let $Y$ be a subset of $C\left(\mathbb{R}_{+}\right)$or of $L_{\text {loc }}^{p}\left(\mathbb{R}_{+}\right)$for some $1 \leq p \leq \infty$.

(1) If $1 \leq p \leq \infty$ and $\Psi: L^{p}\left(\mathbb{R}_{+}\right) \rightarrow L^{p}\left(\mathbb{R}_{+}\right)$is causal, then $\Psi$ extends in a natural way to an operator mapping $L_{\mathrm{loc}}^{p}([0, T))$ into itself: for $v \in L_{\mathrm{loc}}^{p}([0, T))$ simply set

$$
(\Psi(v))(t):=\left(\Psi\left(P_{\tau} v\right)\right)(t), \quad 0 \leq t \leq \tau<T .
$$

By causality of $\Psi$, this definition does not depend on the choice of $\tau$ and so $\Psi(v)$ is a well-defined function in $L_{\mathrm{loc}}^{p}([0, T))$ for any $v \in L_{\mathrm{loc}}^{p}([0, T))$.

(2) If $\Psi: C\left(\mathbb{R}_{+}\right) \rightarrow Y$ is causal and $I$ is an interval of the form $[0, T)$ or $[0, T]$ (where $T<\infty$ ), then $\Psi$ extends in a natural way to an operator mapping on $C(I)$ : for $v \in C(I)$ simply set

$$
(\Psi(v))(t):=\left(\Psi\left(Q_{\tau} v\right)\right)(t), \quad 0 \leq t \leq \tau, \tau \in I .
$$

Again, the causality of $\Psi$ guarantees that this definition does not depend on the choice of $\tau$ and so $\Psi(v)$ is a well-defined function on $Y_{I}$ for any $v \in C(I)$, where $Y_{I}:=C(I)$ if $Y=C\left(\mathbb{R}_{+}\right)$and $Y_{I}:=L_{\mathrm{loc}}^{p}(I)$ if $Y=L_{\mathrm{loc}}^{p}\left(\mathbb{R}_{+}\right)$(of course, in the latter case, if $I$ is compact, then $Y_{I}=L^{p}(I)$ ).

We will not distinguish notationally between the original causal operator and its various extensions. 


\section{Preliminaries}

\subsection{An absolute stability result}

Consider the feedback system shown in Figure 3, where $N$ is a static, possibly time-varying, nonlinearity, the operator $G: L^{2}\left(\mathbb{R}_{+}\right) \rightarrow L^{2}\left(\mathbb{R}_{+}\right)$is linear, bounded and shift-invariant and $r \in L_{\text {loc }}^{2}\left(\mathbb{R}_{+}\right)$is an input signal.

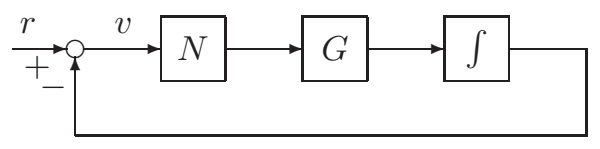

FIGURE 3. Feedback system with static nonlinearity.

The equation describing the system in Figure 3 is

$$
v(t)=r(t)-\int_{0}^{t}(G(N(\cdot, v)))(\tau) \mathrm{d} \tau .
$$

Since shift-invariance implies causality, $G$ can be extended to a shift-invariant operator mapping $L_{\text {loc }}^{2}\left(\mathbb{R}_{+}\right)$into itself (see Rem. 1.1). A global solution of $(2.1)$ is a function $v \in L_{\text {loc }}^{2}\left(\mathbb{R}_{+}\right)$such that $N(\cdot, v) \in L_{\text {loc }}^{2}\left(\mathbb{R}_{+}\right)$and $(2.1)$ is satisfied almost everywhere.

Denoting the transfer function of $G$ by $\mathbf{G}$, we have that $\mathbf{G} \in H^{\infty}\left(\mathbb{C}_{+}\right)$, that is, $\mathbf{G}$ is holomorphic and bounded in the open right-half plane. We assume that

(L) $\mathbf{G}(0):=\lim _{s \rightarrow 0, \operatorname{Re} s>0} \mathbf{G}(s)$ exists, $\quad \mathbf{G}(0)>0 \quad$ and $\limsup _{s \rightarrow 0, \operatorname{Re} s>0}|(\mathbf{G}(s)-\mathbf{G}(0)) / s|<\infty$.

Set

$$
f(G):=\underset{\omega \in \mathbb{R}}{\operatorname{ess} \inf } \operatorname{Re}(\mathbf{G}(i \omega) / i \omega) .
$$

We claim that $-\infty<f(G) \leq 0$. Indeed, since $\mathbf{G}$ is in $H^{\infty}\left(\mathbb{C}_{+}\right)$, we obtain $f(G) \leq 0$ by taking $|\omega| \rightarrow \infty$ in (2.2) and, noting that $\operatorname{Re}(\mathbf{G}(i \omega) / i \omega)=\operatorname{Re}([\mathbf{G}(i \omega)-\mathbf{G}(0)] / i \omega)$, the inequality $f(G)>-\infty$ follows from assumption (L). Hence, the positivity condition

$$
\frac{1}{a}+\underset{\omega \in \mathbb{R}}{\operatorname{ess} \inf } \operatorname{Re}\left(\frac{\mathbf{G}(i \omega)}{i \omega}\right)>0
$$

holds, provided that

$$
a<1 /|f(G)| \quad \text { (with the convention } 1 / 0:=\infty \text { ). }
$$

The following proposition will be employed in the proof of the main results of this paper.

Proposition 2.1. Assume that the following hold:

(a) $G: L^{2}\left(\mathbb{R}_{+}\right) \rightarrow L^{2}\left(\mathbb{R}_{+}\right)$is a linear bounded shift-invariant operator with transfer function $\mathbf{G}$ satisfying assumption $(\mathrm{L})$;

(b) $N: \mathbb{R}_{+} \times \mathbb{R} \rightarrow \mathbb{R}$ is a static nonlinearity, satisfying

$$
0 \leq N(t, \xi) \xi \leq a \xi^{2}, \quad \forall(t, \xi) \in\left[t_{0}, \infty\right) \times \mathbb{R}
$$

for some $0<a<1 /|f(G)|$ and some $t_{0} \geq 0$;

(c) $r \in L^{2}\left(\mathbb{R}_{+}\right)+\mathbb{R} \theta$. 
If $v$ is a global solution of (2.1), then

(i) $v-r \in L^{\infty}\left(\mathbb{R}_{+}\right)$;

(ii) $N(\cdot, v) \in L^{2}\left(\mathbb{R}_{+}\right)$;

(iii) $\int_{0}^{t} N(\tau, v(\tau)) \mathrm{d} \tau$ converges to a finite limit as $t \rightarrow \infty$.

Proposition 2.1 is a special case of Theorem 3.3 in [5], the proof of which relies crucially on the positivity property (2.3).

\subsection{A technical lemma}

A function $u \in C\left(\mathbb{R}_{+}\right)$is called ultimately non-decreasing (non-increasing) if there exists $\tau \in \mathbb{R}_{+}$such that $u$ is non-decreasing (non-increasing) on $[\tau, \infty) ; u$ is said to be approximately ultimately non-decreasing (nonincreasing) if, for all $\varepsilon>0$, there exists an ultimately non-decreasing (non-increasing) function $v \in C\left(\mathbb{R}_{+}\right)$such that

$$
|u(t)-v(t)| \leq \varepsilon, \quad \forall t \in \mathbb{R}_{+} .
$$

The following lemma will be used in Sections 4 and 5 .

Lemma 2.2. Let $g \in W_{\text {loc }}^{1,1}\left(\mathbb{R}_{+}\right)$satisfy

$$
g^{\prime}=f_{1}\left(f_{2}-f_{3}\right)
$$

where $f_{1} \notin L^{1}\left(\mathbb{R}_{+}\right)$is measurable, non-negative and bounded, $f_{2}$ is measurable with $\lim _{t \rightarrow \infty} f_{2}(t)=l \neq 0$ and $f_{3} \in L^{p}\left(\mathbb{R}_{+}\right)$, where $1 \leq p<\infty$. Then the following assertions hold:

(i) if $l<0$, then $g$ is approximately ultimately non-increasing and $\lim _{t \rightarrow \infty} g(t)=-\infty$;

(ii) if $l>0$, then $g$ is approximately ultimately non-decreasing and $\lim _{t \rightarrow \infty} g(t)=+\infty$.

The interpretation of this result is that, under the stated assumptions, $f_{2}$ ultimately "dominates" $f_{3}$.

Proof. Assume that $l<0$. (The case $l>0$ can be treated in analogous way.) Write $f_{3}=h_{1}+h_{2}$, where

$$
h_{1}(t):=\max \left\{f_{3}(t), l / 3\right\}, \quad h_{2}(t):=f_{3}(t)-h_{1}(t), \quad \forall t \in \mathbb{R}_{+} .
$$

Thus, $h_{1}(t) \geq l / 3$ and $h_{2}(t)=\chi_{E}(t)\left(f_{3}(t)-l / 3\right)$ for all $t \in \mathbb{R}_{+}$, where $\chi_{E}$ is the indicator function of the set $E:=\left\{t \in \mathbb{R}_{+}: f_{3}(t)<l / 3\right\}$. In particular, since $f_{3} \in L^{p}\left(\mathbb{R}_{+}\right)$for some $p \in[1, \infty)$ and $l<0$, the set $E$ has finite Lebesgue measure, and so, $h_{2} \in L^{1}\left(\mathbb{R}_{+}\right)$. Since $h_{1} \geq l / 3$ and $\lim _{t \rightarrow \infty} f_{2}(t)=l$, by taking $T \geq 0$ large enough, we have

$$
f_{2}(t)-h_{1}(t) \leq l / 3, \quad \forall t \geq T .
$$

Using non-negativity of $f_{1}$ and (2.6) we obtain from the identity $g^{\prime}=f_{1}\left(f_{2}-h_{1}-h_{2}\right)$ that

$$
g^{\prime}(t) \leq \frac{l}{3} f_{1}(t)-f_{1}(t) h_{2}(t), \quad \text { a.e. } \quad t \geq T .
$$

Choose a sequence $\left(t_{n}\right)$ such that $t_{n} \uparrow \infty$ and $t_{n} \geq T$ for all $n$. Define

$$
g_{n}(t)= \begin{cases}g(t), & 0 \leq t \leq t_{n} \\ g\left(t_{n}\right)+\int_{t_{n}}^{t} f_{1}(\tau)\left(f_{2}(\tau)-h_{1}(\tau)\right) \mathrm{d} \tau, & t>t_{n} .\end{cases}
$$

Then, for all $t \geq t_{n}$,

$$
\left|g(t)-g_{n}(t)\right|=\left|\int_{t_{n}}^{t} f_{1}(\tau) h_{2}(\tau) \mathrm{d} \tau\right| \leq \int_{t_{n}}^{\infty}\left|f_{1}(\tau) h_{2}(\tau)\right| \mathrm{d} \tau
$$


Since $f_{1}$ is bounded and $h_{2} \in L^{1}\left(\mathbb{R}_{+}\right)$, we conclude that $f_{1} h_{2} \in L^{1}\left(\mathbb{R}_{+}\right)$and thus

$$
\sup _{t \geq 0}\left|g(t)-g_{n}(t)\right| \rightarrow 0 \quad \text { as } n \rightarrow \infty .
$$

It follows from (2.6) and (2.8), combined with non-negativity of $f_{1}$, that $g_{n}$ is ultimately non-increasing, showing that $g$ is approximately ultimately non-increasing. Furthermore, by $(2.7)$

$$
g(t)-g(\tau) \leq \frac{l}{3} \int_{T}^{t} f_{1}(\tau) \mathrm{d} \tau-\int_{T}^{t} f_{1}(\tau) h_{2}(\tau) \mathrm{d} \tau, \quad \forall t \geq T .
$$

Since $f_{1} \geq 0, f_{1} \notin L^{1}\left(\mathbb{R}_{+}\right)$and $f_{1} h_{2} \in L^{1}\left(\mathbb{R}_{+}\right)$, it follows that $\lim _{t \rightarrow \infty} g(t)=-\infty$.

\section{A CLASS OF HYSTERESIS OPERATORS}

In this section we define and discuss properties of a class of hysteresis operators. For more information on the mathematical theory of hysteresis operators see, for example, $[2,3,14]$. We remark that our treatment of hysteresis operators has been strongly influenced by Chapter 2 in [3].

A function $f: \mathbb{R}_{+} \rightarrow \mathbb{R}_{+}$is called a time transformation if it is continuous and non-decreasing with $f(0)=0$ and $\lim _{t \rightarrow \infty} f(t)=\infty$; in other words, $f$ is a time transformation if it is continuous, non-decreasing and surjective. An operator $\Phi: C\left(\mathbb{R}_{+}\right) \rightarrow C\left(\mathbb{R}_{+}\right)$is called rate independent if, for every time transformation $f$,

$$
(\Phi(u \circ f))(t)=(\Phi(u))(f(t)), \quad \forall u \in C\left(\mathbb{R}_{+}\right), \forall t \in \mathbb{R}_{+} .
$$

We say that $\Phi: C\left(\mathbb{R}_{+}\right) \rightarrow C\left(\mathbb{R}_{+}\right)$is a hysteresis operator if $\Phi$ is causal and rate independent.

The numerical value set $\operatorname{NVS} \Phi$ of a hysteresis operator $\Phi$ is defined by

$$
\operatorname{NVS} \Phi:=\left\{(\Phi(u))(t): u \in C\left(\mathbb{R}_{+}\right), t \in \mathbb{R}_{+}\right\} .
$$

For $w \in C([0, a])($ with $a \geq 0)$ and $\gamma, \delta>0$, we define

$$
\mathcal{C}(w ; \delta, \gamma):=\left\{v \in C([0, a+\gamma]):\left.v\right|_{[0, a]}=w, \sup _{t \in[a, a+\gamma]}|v(t)-w(a)| \leq \delta\right\} .
$$

Depending on context, we will impose some or all of the following six conditions on hysteresis operators $\Phi$ : $C\left(\mathbb{R}_{+}\right) \rightarrow C\left(\mathbb{R}_{+}\right)$.

(N1) $\Phi\left(W_{\text {loc }}^{1,1}\left(\mathbb{R}_{+}\right)\right) \subset W_{\text {loc }}^{1,1}\left(\mathbb{R}_{+}\right)$.

(N2) $\Phi$ is monotone in the sense that, for all $u \in W_{\text {loc }}^{1,1}\left(\mathbb{R}_{+}\right)$,

$$
(\Phi(u))^{\prime}(t) u^{\prime}(t) \geq 0, \quad \text { a.e. } t \in \mathbb{R}_{+} .
$$

(N3) There exists $\lambda>0$ such that for all $a \geq 0$ and $w \in C([0, a])$, there exist numbers $\gamma, \delta>0$ such that

$$
\max _{\tau \in[a, a+\gamma]}|(\Phi(u))(\tau)-(\Phi(v))(\tau)| \leq \lambda \max _{\tau \in[a, a+\gamma]}|u(\tau)-v(\tau)|, \quad \forall u, v \in \mathcal{C}(w ; \delta, \gamma) .
$$

(N4) For all $a>0$ and all $u \in C([0, a))$, there exists $\beta>0$ such that

$$
\max _{\tau \in[0, t]}|(\Phi(u))(\tau)| \leq \beta\left(1+\max _{\tau \in[0, t]}|u(\tau)|\right), \quad \forall t \in[0, a) .
$$


(N5) If $u \in C\left(\mathbb{R}_{+}\right)$is approximately ultimately non-decreasing and $\lim _{t \rightarrow \infty} u(t)=\infty$, then $(\Phi(u))(t)$ and $(\Phi(-u))(t)$ converge, as $t \rightarrow \infty$, to sup NVS $\Phi$ and $\inf \operatorname{NVS} \Phi$, respectively.

(N6) If, for $u \in C\left(\mathbb{R}_{+}\right), \lim _{t \rightarrow \infty}(\Phi(u))(t) \in \operatorname{int} \operatorname{NVS} \Phi$, then $u$ is bounded.

Note that, in (N3) and (N4), the functions $\Phi(u)$ and $\Phi(v)$ are well-defined by Remark 1.1. It is not difficult to deduce that (N5) implies that NVS $\Phi$ is an interval. As is well-known (see, for example, [3,16]), many hysteresis operators are Lipschitz continuous in the sense that, for some $\lambda>0$,

$$
\sup _{\tau \in \mathbb{R}_{+}}|(\Phi(u))(\tau)-(\Phi(v))(\tau)| \leq \lambda \sup _{\tau \in \mathbb{R}_{+}}|u(\tau)-v(\tau)|, \quad \forall u, v \in C\left(\mathbb{R}_{+}\right),
$$

in which case (N3) is trivially satisfied. An important consequence of assumptions (N1)-(N3) is described in the following lemma, a proof of which can be found in [16].

Lemma 3.1. Let $\Phi: C\left(\mathbb{R}_{+}\right) \rightarrow C\left(\mathbb{R}_{+}\right)$be a hysteresis operator satisfying (N1)-(N3). Then, for any $u \in$ $W_{\text {loc }}^{1,1}\left(\mathbb{R}_{+}\right)$, there exists a measurable function $d_{u}: \mathbb{R}_{+} \rightarrow[0, \lambda]$ such that

$$
(\Phi(u))^{\prime}(t)=d_{u}(t) u^{\prime}(t), \quad \text { a.e. } t \in \mathbb{R}_{+} .
$$

Remark 3.2. An important and well-known property of hysteresis operators is that they commute with $Q_{\tau}$ for all $\tau \in \mathbb{R}_{+}$, that is, if $\Phi$ is a hysteresis operator, then

$$
\Phi Q_{\tau}=Q_{\tau} \Phi, \forall \tau \in \mathbb{R}_{+},
$$

where $Q_{\tau}: C\left(\mathbb{R}_{+}\right) \rightarrow C\left(\mathbb{R}_{+}\right)$is given by (1.1). This commutativity property is an easy consequence of causality and rate-independence.

There exist causal operators $\Phi: C\left(\mathbb{R}_{+}\right) \rightarrow C\left(\mathbb{R}_{+}\right)$satisfying (3.3), but which are not hysteresis operators. For example, consider the operator $\Phi: C\left(\mathbb{R}_{+}\right) \rightarrow C\left(\mathbb{R}_{+}\right)$defined by

$$
(\Phi(u))(t)=(1+\varphi(t)) u(t)-\int_{0}^{t} \varphi^{\prime}(\tau) u(\tau) \mathrm{d} \tau, \quad \forall t \in \mathbb{R}_{+},
$$

where $\varphi: \mathbb{R}_{+} \rightarrow \mathbb{R}$ is continuously differentiable. Clearly, $\Phi$ is causal and a routine calculation shows that $\Phi$ satisfies (3.3). However, unless $\varphi$ is constant, $\Phi$ is not, in general, rate-independent and hence not a hysteresis operator.

In fact, it is the commutativity property, rather than rate independence, which is used in the proof of Lemma 3.1 and, consequently, in Sections 4-6. Therefore, in the results of Sections 4-6, the requirement that $\Phi$ be a hysteresis operator can be weakened to the assumption that $\Phi$ is causal and satisfies (3.3). However, we believe that non-rate-independent causal operators satisfying (3.3) may be of limited physical relevance and so are mainly of academic interest. For this reason, we assume that the operators under consideration are hysteresis (i.e., causal and rate-independent) rather than causal operators satisfying (3.3).

Remark 3.3. Assumption (N3), causality and rate-independence imply the following stronger assertion:

(N3') There exists $\lambda>0$ such that for all $a \geq 0$ and $w \in C([0, a])$, there exist numbers $\gamma, \delta>0$ such that

$$
\max _{\tau \in[a, a+\varepsilon]}|(\Phi(u))(\tau)-(\Phi(v))(\tau)| \leq \lambda \max _{\tau \in[a, a+\varepsilon]}|u(\tau)-v(\tau)|, \quad \forall u, v \in \mathcal{C}(w ; \delta, \gamma), \quad \forall \varepsilon \in(0, \gamma] .
$$

Indeed, let us take $\lambda$ from (3.2) and arbitrary $\varepsilon \in(0, \gamma]$. Invoking the operator $Q_{\tau}$ (with $a<\tau \leq a+\gamma$ ) defined in (1.1)), we see that inequality (3.4) is equivalent to

$$
\max _{\tau \in[a, a+\gamma]}\left|\left(Q_{a+\varepsilon} \Phi(u)\right)(\tau)-\left(Q_{a+\varepsilon} \Phi(v)\right)(\tau)\right| \leq \lambda \max _{\tau \in[a, a+\gamma]}\left|\left(Q_{a+\varepsilon} u\right)(\tau)-\left(Q_{a+\varepsilon} v\right)(\tau)\right| .
$$


The latter inequality is a simple consequence of (N3) and (3.3).

The set of all hysteresis operators satisfying (N1)-(N6) is denoted by $\mathcal{N}(\lambda)$, where $\lambda>0$ is the constant associated with (N3). It is well known that many standard hysteresis nonlinearities which are important in control engineering are contained in $\mathcal{N}(\lambda)$ for some suitable $\lambda>0$ : two examples are given below and others can be found in $[16,19]$.

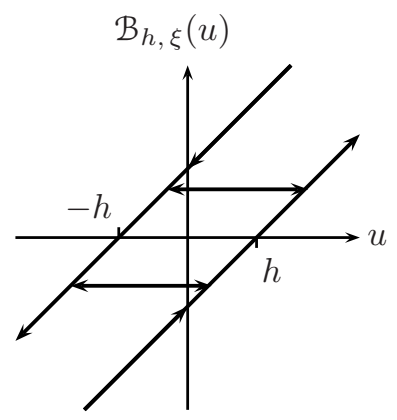

FiguRE 4. Backlash hysteresis.

Backlash hysteresis (play operator). A discussion of the backlash operator (also called play operator) can be found in a number of references, see for example [3,14] and [16]. Let $h \in \mathbb{R}_{+}$and introduce the function

$$
b_{h}: \mathbb{R}^{2} \rightarrow \mathbb{R}, \quad(v, w) \mapsto \max \{v-h, \min \{v+h, w\}\}= \begin{cases}v-h, & w<v-h \\ w, & w \in[v-h, v+h] \\ v+h, & w>v+h\end{cases}
$$

Let $C_{\mathrm{pm}}\left(\mathbb{R}_{+}\right)$denote the space of continuous piecewise monotone functions defined on $\mathbb{R}_{+}$. For all $h \in \mathbb{R}_{+}$and all $\xi \in \mathbb{R}$, we define the operator $\mathcal{B}_{h, \xi}: C_{\mathrm{pm}}\left(\mathbb{R}_{+}\right) \rightarrow C\left(\mathbb{R}_{+}\right)$by

$$
\left(\mathcal{B}_{h, \xi}(u)\right)(t)= \begin{cases}b_{h}(u(0), \xi) & \text { for } t=0 \\ b_{h}\left(u(t),\left(\mathcal{B}_{h, \xi}(u)\right)\left(t_{i}\right)\right) & \text { for } t_{i}<t \leq t_{i+1}, i=0,1,2, \ldots,\end{cases}
$$

where $0=t_{0}<t_{1}<t_{2}<\ldots, \lim _{n \rightarrow \infty} t_{n}=\infty, u$ is monotone on each interval $\left[t_{i}, t_{i+1}\right]$, and $\xi$ plays the role of an "initial state". It is not difficult to show that the definition is independent of the choice of the partition $\left(t_{i}\right)$. Figure 4 illustrates how $\mathcal{B}_{h, \xi}$ acts.

It is well-known that $\mathcal{B}_{h, \xi}$ extends to a Lipschitz continuous operator on $C\left(\mathbb{R}_{+}\right)$(with Lipschitz constant 1), the so-called backlash operator, which we will denote by the same symbol $\mathcal{B}_{h, \xi}$. It is also well-known (and easy to check) that $\mathcal{B}_{h, \xi}$ is a hysteresis operator. As shown in [16] for example, $\mathcal{B}_{h, \xi}$ satisfies (N1) $-(\mathrm{N} 6)$ (with $\lambda=1$ in $(\mathrm{N} 3)$ ). It is obvious that $\operatorname{NVSB}_{h, \xi}=\mathbb{R}$.

Prandtl operators. The Prandtl operator (also called the Prandtl-Ishlinskii operator), introduced below, is a generalization of the backlash operator. For certain input functions, it exhibits nested loops in the corresponding input-output characteristics. Let $\xi$ be a Preisach memory curve, i.e. $\xi: \mathbb{R}_{+} \rightarrow \mathbb{R}$ is a function which has compact support and is globally Lipschitz with Lipschitz constant 1 . Furthermore, let $\mu$ be a signed Borel measure on $\mathbb{R}_{+}$such that $|\mu|(K)<\infty$ for all compact sets $K \subset \mathbb{R}_{+}$, where $|\mu|$ denotes the total variation of $\mu$. The operator $\mathcal{P}_{\xi}: C\left(\mathbb{R}_{+}\right) \rightarrow C\left(\mathbb{R}_{+}\right)$defined by

$$
\left(\mathcal{P}_{\xi}(u)\right)(t)=\int_{0}^{\infty}\left(\mathcal{B}_{h, \xi(h)}(u)\right)(t) \mathrm{d} \mu(h), \quad \forall u \in C\left(\mathbb{R}_{+}\right), \forall t \in \mathbb{R}_{+}
$$


is called a Prandtl operator, $c f$. [3], p. 54. It is well-known (and easy to check) that $\mathcal{P}_{\xi}$ is a hysteresis operator. Assume that the measure $\mu$ is finite. Then the operator $\mathcal{P}_{\xi}$ is Lipschitz continuous with Lipschitz constant $|\mu|\left(\mathbb{R}_{+}\right)$(since the backlash operator is Lipschitz continuous with Lipschitz constant 1). Furthermore, if we additionally assume that $\mu$ is positive, then, as shown for example in [16], (N1)-(N6) hold (with $\lambda=\mu\left(\mathbb{R}_{+}\right)$ in $(\mathrm{N} 3))$. For $\xi \equiv 0$ and the measure $\mu$ given by $\mu(E)=\int_{E}(\sin (\pi h)+1) \chi_{[0,10]} d h$ (where $\chi_{[0,10]}$ denotes the indicator function of the interval $[0,10]$ ), the Prandtl operator is illustrated in Figure 5.
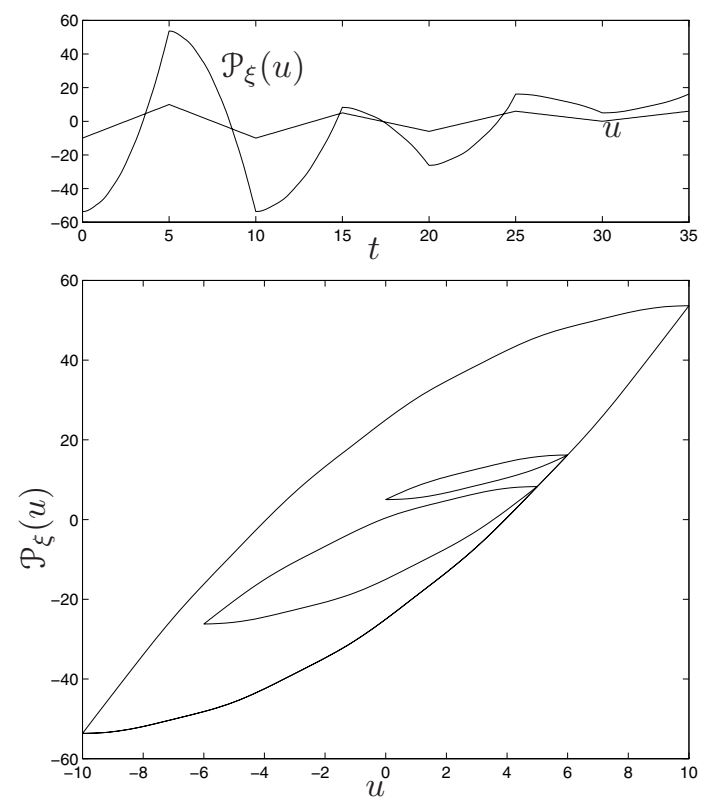

Figure 5. Example of Prandtl hysteresis.

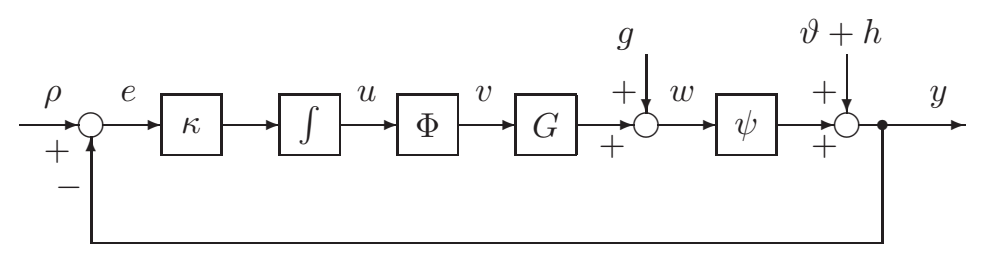

FIGURE 6. Integral control in the presence of input hysteresis.

It follows from [16] that $\operatorname{NVSP}_{\xi}=\mathbb{R}$, provided that $\mu \neq 0$. Finally, we remark that the above class of Prandtl operators can be generalized to include the so-called Preisach operators (see [3]): a large class of such operators also satisfy (N1)-(N6) (see [16]).

\section{LOW-GAIN INTEGRAL CONTROL IN THE PRESENCE OF INPUT HYSTERESIS}

Consider the feedback system shown in Figure 6 , where $\rho \in \mathbb{R}$ is a constant, $\kappa: \mathbb{R}_{+} \rightarrow \mathbb{R}$ is a time-varying gain, the operator $G: L^{2}\left(\mathbb{R}_{+}\right) \rightarrow L^{2}\left(\mathbb{R}_{+}\right)$is linear, bounded and shift-invariant, $\Phi$ is a hysteresis operator, 
$\psi: \mathbb{R} \rightarrow \mathbb{R}$ is non-decreasing and globally Lipschitz, the function $g \in L^{2}\left(\mathbb{R}_{+}\right)$models the effect of non-zero initial conditions of the system with input-output operator $G$ and $\vartheta+h$ is a disturbance containing a constant $\vartheta$ and a locally integrable function $h$.

This system is described by the following functional differential equation:

$$
u^{\prime}=\kappa(\rho-\vartheta-h-\psi(g+(G \circ \Phi)(u))), \quad u(0)=u^{0} \in \mathbb{R} .
$$

A solution of (4.1) on the interval $[0, \tau)$ is a function $u \in W_{\text {loc }}^{1,1}([0, \tau))$ such that $u(0)=u^{0}$ and the differential equation in (4.1) is satisfied almost everywhere on $[0, \tau)$. Recall that, by causality, the operator $G \circ \Phi$ is well-defined on $W_{\text {loc }}^{1,1}([0, \tau))$, see Remark 1.1.

Our objective is to determine gain functions $\kappa$ such that the tracking error

$$
e(t):=\rho-y(t)=\rho-\vartheta-h(t)-\psi(g(t)+((G \circ \Phi)(u))(t))
$$

becomes small in a certain sense as $t \rightarrow \infty$. For example, we might want to achieve "tracking in measure", i.e., for every $\varepsilon>0$, the Lebesgue measure of the set $\{\tau \geq t:|e(\tau)| \geq \varepsilon\}$ tends to 0 as $t \rightarrow \infty$, or the aim might be "asymptotic tracking", that is $\lim _{t \rightarrow \infty} e(t)=0$. Trivially, tracking in measure is guaranteed if $e$ is of the form $e=e_{1}+e_{2}$, where $\lim _{t \rightarrow \infty} e_{1}(t)=0$ and $e_{2} \in L^{p}\left(\mathbb{R}_{+}\right)$for some $p \in[1, \infty)$.

The generality of the input and output nonlinearities $\Phi$ and $\psi$ allows specific cases wherein tracking of all constant reference signals $\rho$ and rejection of all constant disturbances $\vartheta$ may not be feasible. For this reason, we impose a restriction on the difference $\rho-\vartheta$; namely, it should belong to the following set:

$$
\mathcal{R}(G, \Phi, \psi):=\{\psi(\mathbf{G}(0) v): v \in \overline{\mathrm{NVS}} \Phi\} .
$$

The intuition underlying $\mathcal{R}(G, \Phi, \psi)$ is as follows. If asymptotic tracking occurs, we would expect that $\Phi^{\infty}:=$ $\lim _{t \rightarrow \infty}(\Phi(u))(t)$ exists. Assuming that $\Phi^{\infty}$ is finite and that the final-value theorem holds for the linear system with input-output operator $G$, we may conclude that $\lim _{t \rightarrow \infty}(G \circ \Phi(u))(t)=\mathbf{G}(0) \Phi^{\infty}$. If, additionally, $\lim _{t \rightarrow \infty} g(t)=\lim _{t \rightarrow \infty} h(t)=0$, it follows from (4.2) that $\rho-\vartheta \in \mathcal{R}(G, \Phi, \psi)$. In fact, it has been shown in [6] that in the case of static input nonlinearities, if $\psi$ is continuous and monotone, then $\rho-\vartheta \in \mathcal{R}(G, \Phi, \psi)$ is close to being a necessary condition for asymptotic tracking.

Theorem 4.1. Assume that the following hold:

(a) $G: L^{2}\left(\mathbb{R}_{+}\right) \rightarrow L^{2}\left(\mathbb{R}_{+}\right)$is a linear bounded shift-invariant operator with transfer function $\mathbf{G}$, satisfying assumption $(\mathrm{L})$;

(b) $g \in L^{2}\left(\mathbb{R}_{+}\right)$;

(c) $\Phi \in \mathcal{N}\left(\lambda_{1}\right)$;

(d) $\psi: \mathbb{R} \rightarrow \mathbb{R}$ is non-decreasing and globally Lipschitz with Lipschitz constant $\lambda_{2}$;

(e) $\rho-\vartheta \in \mathcal{R}(G, \Phi, \psi)$;

(f) $h$ is such that $h \in L^{1}\left(\mathbb{R}_{+}\right) \cap L^{2}\left(\mathbb{R}_{+}\right)$and the function $t \mapsto \int_{t}^{\infty}|h(\tau)| \mathrm{d} \tau$ is in $L^{2}\left(\mathbb{R}_{+}\right)$;

(g) $\kappa: \mathbb{R}_{+} \rightarrow \mathbb{R}$ is measurable, non-negative and bounded with

$$
\limsup _{t \rightarrow \infty} \kappa(t)<\frac{1}{\lambda_{1} \lambda_{2}|f(G)|},
$$

where $1 / 0:=\infty$.

Then there exists a unique solution $u \in W_{\mathrm{loc}}^{1,1}\left(\mathbb{R}_{+}\right)$of (4.1) and the following statements hold:

(i) $(\Phi(u))^{\prime} \in L^{2}\left(\mathbb{R}_{+}\right)$and the limit $\Phi^{\infty}:=\lim _{t \rightarrow \infty}(\Phi(u))(t)$ exists and is finite. 
(ii) The signals $w=g+(G \circ \Phi)(u)$ and $y=\psi(w)+\vartheta+h$ (see Fig. 6) can be decomposed as $w=w_{1}+w_{2}$ and $y=y_{1}+y_{2}$, where $w_{1}, y_{1}$ are continuous and have finite limits

$$
w_{1}^{\infty}:=\lim _{t \rightarrow \infty} w_{1}(t)=\mathbf{G}(0) \Phi^{\infty}, \quad y_{1}^{\infty}:=\lim _{t \rightarrow \infty} y_{1}(t)=\psi\left(\mathbf{G}(0) \Phi^{\infty}\right)+\vartheta,
$$

and $w_{2}, y_{2} \in L^{2}\left(\mathbb{R}_{+}\right)$. Under the additional assumptions that

$$
\lim _{t \rightarrow \infty}(g(t)+(\Phi(u))(0)((G \theta)(t)-\mathbf{G}(0)))=0
$$

and

we have

$$
\lim _{t \rightarrow \infty} h(t)=0
$$

$$
\lim _{t \rightarrow \infty} w_{2}(t)=0, \quad \lim _{t \rightarrow \infty} y_{2}(t)=0 .
$$

(iii) If $\kappa \notin L^{1}\left(\mathbb{R}_{+}\right)$, then $y_{1}^{\infty}=\lim _{t \rightarrow \infty} y_{1}(t)=\rho$ and the error signal $e=\rho-y$ can be decomposed as $e=e_{1}+e_{2}$, where $e_{1}$ is continuous with $\lim _{t \rightarrow \infty} e_{1}(t)=0$ and $e_{2} \in L^{2}\left(\mathbb{R}_{+}\right)$. If, additionally, (4.3) and (4.4) hold, then

$$
\lim _{t \rightarrow \infty} e(t)=0
$$

(iv) If $\rho-\vartheta$ is an interior point of the set $\mathcal{R}(G, \Phi, \psi)$, then $u$ is bounded.

Remark 4.2. (1) Statement (iii) of Theorem 4.1 implies tracking in measure and, moreover, guarantees asymptotic tracking, provided that (4.3) and (4.4) hold. Trivially, if $\lim _{t \rightarrow \infty} g(t)=0$ and

$$
\lim _{t \rightarrow \infty}(G \theta)(t)=\mathbf{G}(0),
$$

then (4.3) is satisfied. If the impulse response of $G$ is a finite Borel measure $\mu$, for example, if

$$
\mu(\mathrm{d} \tau)=f_{a}(\tau) \mathrm{d} \tau+\sum_{i=0}^{\infty} f_{i} \delta_{t_{i}}(\mathrm{~d} \tau)
$$

where $f_{a} \in L^{1}\left(\mathbb{R}_{+}\right),\left\{f_{i}\right\} \in l^{1}\left(\mathbb{Z}_{+}\right)$and $t_{i} \geq 0$, then (4.5) holds. Finally, since it follows from assumption (L) via the Paley-Wiener theorem that $G \theta-\mathbf{G}(0) \theta \in L^{2}\left(\mathbb{R}_{+}\right)$, we conclude that if the limit on the left-hand side of (4.5) exists, then it must be equal to $\mathbf{G}(0)$.

(2) In general, $\vartheta$ is unknown, but it is reasonable to assume that $\vartheta \in\left[\vartheta_{1}, \vartheta_{2}\right]$, where $\vartheta_{1}$ and $\vartheta_{2}$ are known constants. The condition $\rho-\vartheta_{1}, \rho-\vartheta_{2} \in \mathcal{R}(G, \Phi, \psi)$ does not involve $\vartheta$ and is sufficient for assumption (e) to hold.

(3) Note that it is not necessary to know $f(G)$ or the constants $\lambda_{1}, \lambda_{2}$ from (c) and (d), respectively, in order to apply Theorem 4.1. If $\kappa$ is chosen such that $\kappa(t) \rightarrow 0$ and $\kappa \notin L^{1}\left(\mathbb{R}_{+}\right)\left(e . g ., \kappa(t)=(1+t)^{-p}\right.$ with $p \in(0,1])$, then the conclusions of statement (iii) hold. However, from a practical point of view, gain functions $\kappa$ with $\lim _{t \rightarrow \infty} \kappa(t)=0$ might not be appropriate, since, in that case, the system essentially operates in open loop as $t \rightarrow \infty$. In [20] it has been shown how $|f(G)|$ (or upper bounds for $|f(G)|$ ) can be obtained from frequency-response experiments performed on the linear part of the plant.

(4) Assumption (f) is satisfied if there exists $\alpha>1$ such that the function $t \mapsto(1+t)^{\alpha} h(t)$ is in $L^{2}\left(\mathbb{R}_{+}\right)$.

Proof of Theorem 4.1. Let $u$ be the unique solution of (4.1) defined on $\mathbb{R}_{+}$, the existence of which is proved in Theorem 7.3 of the Appendix.

(i) The key idea is to apply Proposition 2.1 to the signal

$$
w:=g+(G \circ \Phi)(u),
$$


modified by an offset which depends on $\rho$ and $\vartheta$. Since, by assumption (e), $\rho-\vartheta \in \mathcal{R}(G, \Phi, \psi)$, there exists $\Phi^{\sharp} \in \overline{\text { NVS }} \Phi$ satisfying

We define

$$
\psi\left(\mathbf{G}(0) \Phi^{\sharp}\right)=\rho-\vartheta .
$$

$$
\begin{aligned}
\tilde{w} & :=w-\mathbf{G}(0) \Phi^{\sharp}=g+(G \circ \Phi)(u)-\mathbf{G}(0) \Phi^{\sharp}, \\
\tilde{\psi}(\xi) & :=\psi\left(\xi+\mathbf{G}(0) \Phi^{\sharp}\right)-\rho+\vartheta, \quad \forall \xi \in \mathbb{R} .
\end{aligned}
$$

By Lemma 3.1, we have

$$
(\Phi(u))^{\prime}(t)=d_{u}(t) u^{\prime}(t)
$$

where $d_{u}: \mathbb{R}_{+} \rightarrow\left[0, \lambda_{1}\right]$ is measurable.

It follows from (4.1) and (4.6) that $u$ satisfies

$$
(\Phi(u))^{\prime}=-N(\cdot, \tilde{w})-\kappa d_{u} h,
$$

where $N: \mathbb{R}_{+} \times \mathbb{R} \rightarrow \mathbb{R}$ is given by

$$
N(t, \xi):=\kappa(t) d_{u}(t) \tilde{\psi}(\xi)
$$

From (4.7), we infer

where

$$
(\Phi(u))(t)=(\Phi(u))(0)-\int_{0}^{t} N(\tau, \tilde{w}(\tau)) \mathrm{d} \tau+f(t),
$$

$$
f(t):=-\int_{0}^{t} \kappa(\tau) d_{u}(\tau) h(\tau) \mathrm{d} \tau, \quad \forall t \in \mathbb{R}_{+} .
$$

By shift-invariance, $G$ commutes with integration, and thus

$$
((G \circ \Phi)(u))(t)=(\Phi(u))(0)(G \theta)(t)-\int_{0}^{t}(G(N(\cdot, \tilde{w})))(\tau) \mathrm{d} \tau+(G f)(t) .
$$

By adding $g-\mathbf{G}(0) \Phi^{\sharp}$ to both sides of the above identity, we see that $\tilde{w}$ solves an equation of the form (2.1)

$$
\tilde{w}(t)=r(t)-\int_{0}^{t}(G(N(\cdot, \tilde{w})))(\tau) \mathrm{d} \tau,
$$

where

$$
r:=g-\mathbf{G}(0) \Phi^{\sharp} \theta+(\Phi(u))(0) G \theta+G f .
$$

We observe that, by assumption (a) and the Paley-Wiener theorem, $G \theta \in L^{2}\left(\mathbb{R}_{+}\right)+\mathbb{R} \theta$. Consequently, to prove that $r \in L^{2}\left(\mathbb{R}_{+}\right)+\mathbb{R} \theta$, it suffices to show that $f \in L^{2}\left(\mathbb{R}_{+}\right)+\mathbb{R} \theta$. Since $h \in L^{1}\left(\mathbb{R}_{+}\right)$and $\kappa, d_{u}$ are bounded, it follows that $\kappa d_{u} h \in L^{1}\left(\mathbb{R}_{+}\right)$. Thus

$$
f(t)=-\int_{0}^{\infty} \kappa(\tau) \mathrm{d}_{u}(\tau) h(\tau) \mathrm{d} \tau+\int_{t}^{\infty} \kappa(\tau) \mathrm{d}_{u}(\tau) h(\tau) \mathrm{d} \tau
$$

provides the desired decomposition of $f$, since, by assumption, the function $t \mapsto \int_{t}^{\infty}|h(\tau)| \mathrm{d} \tau$ is in $L^{2}\left(\mathbb{R}_{+}\right)$. Hence

Note that

$$
r \in L^{2}\left(\mathbb{R}_{+}\right)+\mathbb{R} \theta
$$

$$
0 \leq N(t, \xi) \xi \leq \lambda_{1} \lambda_{2} \kappa(t) \xi^{2}, \quad \forall(t, \xi) \in \mathbb{R}_{+} \times \mathbb{R} .
$$

By assumption (g) on $\kappa$, there exist $0<a<1 /|f(G)|$ and $t_{0} \geq 0$ such that

$$
0 \leq N(t, \xi) \xi \leq a \xi^{2}, \quad \forall(t, \xi) \in\left[t_{0}, \infty\right) \times \mathbb{R} .
$$


Applying Proposition 2.1 to (4.8), we obtain that $N(\cdot, \tilde{w}) \in L^{2}\left(\mathbb{R}_{+}\right)$and the limit of $\int_{0}^{t} N(\tau, \tilde{w}(\tau)) \mathrm{d} \tau$ as $t \rightarrow \infty$ exists and is finite. Therefore,

and

$$
(\Phi(u))^{\prime}=-N(\cdot, \tilde{w})-\kappa d_{u} h \in L^{2}\left(\mathbb{R}_{+}\right),
$$

$$
\lim _{t \rightarrow \infty}(\Phi(u))(t)=(\Phi(u))(0)-\int_{0}^{\infty} \kappa(\tau) d_{u}(\tau) h(\tau) \mathrm{d} \tau-\lim _{t \rightarrow \infty} \int_{0}^{t} N(\tau, \tilde{w}(\tau)) \mathrm{d} \tau
$$

exists and is finite, which proves statement (i) of Theorem 4.1.

(ii) Define an operator $H$ on $L_{\text {loc }}^{2}\left(\mathbb{R}_{+}\right)$by setting

$$
(H v)(t):=\int_{0}^{t}(G v(\tau)-\mathbf{G}(0) v(\tau)) \mathrm{d} \tau .
$$

Since the transfer function of $H$ is $s \mapsto(\mathbf{G}(s)-\mathbf{G}(0)) / s$ and hence belongs to $H^{\infty}\left(\mathbb{C}_{+}\right)$via assumption (a), we deduce that $H$ is a bounded linear shift-invariant operator on $L^{2}\left(\mathbb{R}_{+}\right)$.

A routine calculation shows that

$$
w=g+(G \circ \Phi)(u)=w_{1}+w_{2},
$$

where

$$
w_{1}:=\mathbf{G}(0) \Phi(u), \quad w_{2}:=g+(\Phi(u))(0)(G \theta-\mathbf{G}(0))+H\left((\Phi(u))^{\prime}\right) .
$$

From statement (i) it follows that $w_{1}^{\infty}=\lim _{t \rightarrow \infty} w_{1}(t)=\mathbf{G}(0) \Phi^{\infty}$. It is also clear that $w_{2} \in L^{2}\left(\mathbb{R}_{+}\right)$due to assumption (b) and the facts that $G \theta-\mathbf{G}(0) \in L^{2}\left(\mathbb{R}_{+}\right)$(by assumption (a) and the Paley-Wiener theorem), $(\Phi(u))^{\prime} \in L^{2}\left(\mathbb{R}_{+}\right)$and $H\left(L^{2}\left(\mathbb{R}_{+}\right)\right) \subset L^{2}\left(\mathbb{R}_{+}\right)$.

We obtain the required decomposition of $y$ by defining

$$
y_{1}:=\psi\left(w_{1}\right)+\vartheta, \quad y_{2}:=y-y_{1}=\psi(w)+h-\psi\left(w_{1}\right) .
$$

Then $y_{1}$ is continuous with $y_{1}^{\infty}=\lim _{t \rightarrow \infty} y_{1}(t)=\psi\left(\mathbf{G}(0) \Phi^{\infty}\right)+\vartheta$. Furthermore, $y_{2} \in L^{2}\left(\mathbb{R}_{+}\right)$since

$$
\left|y_{2}(t)\right| \leq \lambda_{2}\left|w(t)-w_{1}(t)\right|+|h(t)|=\lambda_{2}\left|w_{2}(t)\right|+|h(t)|,
$$

and $w_{2} \in L^{2}\left(\mathbb{R}_{+}\right), h \in L^{2}\left(\mathbb{R}_{+}\right)$.

Let us show that $\lim _{t \rightarrow \infty} H\left((\Phi(u))^{\prime}\right)(t)=0$. Since the transfer function of $H$ also belongs to $H^{2}\left(\mathbb{C}_{+}\right)$ via assumption (a), the convolution kernel $\xi$ of $H$ is in $L^{2}\left(\mathbb{R}_{+}\right)$due to the Paley-Wiener theorem. Thus $H\left((\Phi(u))^{\prime}\right)=\xi *(\Phi(u))^{\prime}$ is a convolution of two $L^{2}\left(\mathbb{R}_{+}\right)$functions, which is known to have a zero limit at infinity. Hence the additional assumptions (4.3) and (4.4) imply $\lim _{t \rightarrow \infty} w_{2}(t)=\lim _{t \rightarrow \infty} y_{2}(t)=0$, completing the proof of statement (ii).

(iii) We use the decomposition $y=y_{1}+y_{2}$ given by (4.11). Seeking a contradiction, suppose that $y_{1}^{\infty}>\rho$ (the case $y_{1}^{\infty}<\rho$ can be treated in an analogous way). Recall the relationship $u^{\prime}=\kappa\left(\rho-y_{1}-y_{2}\right)$. By Lemma 2.2 with $g=u, f_{1}=\kappa, f_{2}=\rho-y_{1}, f_{3}=y_{2}$, we conclude that $u$ is approximately ultimately non-increasing and $\lim _{t \rightarrow \infty} u(t)=-\infty$. Consequently, due to (N5),

$$
\Phi^{\infty}:=\lim _{t \rightarrow \infty}(\Phi(u))(t)=\inf \operatorname{NVS} \Phi .
$$

Since $\rho-\vartheta \in \mathcal{R}(G, \Phi, \psi)$ (by assumption) and using the fact that $\psi$ is non-decreasing and $\mathbf{G}(0)>0$, we obtain

$$
\rho \geq \inf \mathcal{R}(G, \Phi, \psi)+\vartheta=\psi\left(\mathbf{G}(0) \Phi^{\infty}\right)+\vartheta=y_{1}^{\infty},
$$


contradicting the supposition that $y_{1}^{\infty}>\rho$.

To complete the proof of statement (iii), we define $e_{1}:=\rho-y_{1}$ and $e_{2}:=-y_{2}$ and obtain the decomposition $e=\rho-y=e_{1}+e_{2}$. It follows immediately from the properties of $y_{1}$ and $y_{2}$ that $e_{1}$ is continuous with $\lim _{t \rightarrow \infty} e_{1}(t)=0, e_{2} \in L^{2}\left(\mathbb{R}_{+}\right)$. Under additional assumptions (4.3) and (4.4), $\lim _{t \rightarrow \infty} e(t)=0$.

(iv) Assume that $\rho-\vartheta$ is an interior point of $\mathcal{R}(G, \Phi, \psi)$. Let $w_{1}, w_{2}, y_{1}$ and $y_{2}$ be defined by (4.10) and (4.11). By statement (ii), $y_{1}^{\infty}=\psi\left(\mathbf{G}(0) \Phi^{\infty}\right)+\vartheta$. If $\kappa \notin L^{1}\left(\mathbb{R}_{+}\right.$), then $y_{1}^{\infty}=\rho$ (due to statement (iii)) and so $\rho-\vartheta=\psi\left(\mathbf{G}(0) \Phi^{\infty}\right)$. Since $\psi$ is non-decreasing, NVS $\Phi$ is an interval and $\mathbf{G}(0)>0$, we conclude that $\Phi^{\infty}$ is an interior point of NVS $\Phi$. Therefore, by (N6), $u$ is bounded. Finally, if $\kappa \in L^{1}\left(\mathbb{R}_{+}\right)$, we write (4.1) in the form

$$
u^{\prime}=\kappa\left(\rho-y_{1}-y_{2}\right) \text {. }
$$

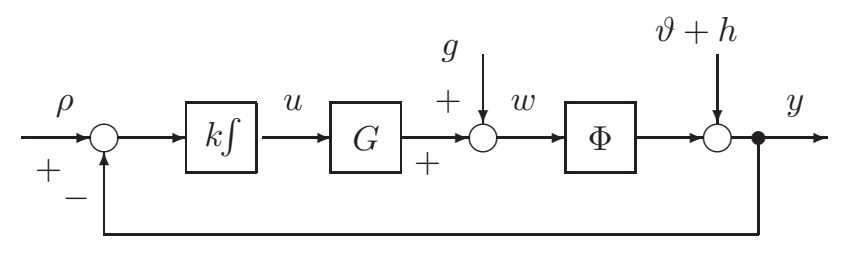

FiguRE 7. Integral control in the presence of output hysteresis.

Note that, since $\kappa$ is bounded, $\kappa$ is also in $L^{2}\left(\mathbb{R}_{+}\right)$. Furthermore, by statement (ii), $y_{1}$ is bounded and $y_{2} \in$ $L^{2}\left(\mathbb{R}_{+}\right)$. Therefore, $\kappa\left(\rho-y_{1}-y_{2}\right) \in L^{1}\left(\mathbb{R}_{+}\right)$, showing that $u^{\prime}$ is integrable and hence $u$ is bounded.

\section{LOW-GAIN INTEGRAL CONTROL IN THE PRESENCE OF OUTPUT HYSTERESiS}

Consider the feedback system shown in Figure 7, where $\rho \in \mathbb{R}$ is a constant, $k \in \mathbb{R}$ is a constant gain, the operator $G: L^{2}\left(\mathbb{R}_{+}\right) \rightarrow L^{2}\left(\mathbb{R}_{+}\right)$is linear, bounded and shift-invariant, $\Phi$ is a hysteresis operator, the function $g$ models the effect of initial conditions of the system with input-output operator $G$ and $\vartheta+h$ is a disturbance containing a constant $\vartheta$ and a locally integrable function $h$.

This system is described by the following functional differential equation:

$$
u^{\prime}=k(\rho-\vartheta-h-\Phi(g+G u)), \quad u(0)=u^{0} \in \mathbb{R} .
$$

A solution of (5.1) on the interval $[0, \tau)$ is a function $u \in W_{\text {loc }}^{1,1}([0, \tau))$ such that $g+G u \in C([0, \tau)), u(0)=u^{0}$ and the differential equation in (5.1) is satisfied almost everywhere on $[0, \tau)$. Recall that, by causality, the function $\Phi(g+G u)$ is well-defined for every $u \in W_{\text {loc }}^{1,1}([0, \tau))$ such that $g+G u \in C([0, \tau))$, see Remark 1.1.

Our objective is to determine gain constants $k$ such that the tracking error

$$
e(t):=\rho-y(t)=\rho-\vartheta-h(t)-(\Phi(g+G u))(t)
$$

converges to zero as $t \rightarrow \infty$.

Theorem 5.1. Assume that the following hold:

(a) $G: L^{2}\left(\mathbb{R}_{+}\right) \rightarrow L^{2}\left(\mathbb{R}_{+}\right)$is a bounded convolution operator with kernel in $L^{2}\left(\mathbb{R}_{+}\right)+\mathbb{R} \delta_{0}$ and transfer function $\mathbf{G}$ satisfying assumption $(\mathrm{L})$; 
(b) $g \in W_{\mathrm{loc}}^{1,1}\left(\mathbb{R}_{+}\right)$and $g^{\prime} \in L^{2}\left(\mathbb{R}_{+}\right)$;

(c) $\Phi \in \mathcal{N}(\lambda)$;

(d) $\rho-\vartheta \in \overline{\mathrm{NVS}} \Phi$;

(e) $h \in L^{2}\left(\mathbb{R}_{+}\right)$and $\lim _{t \rightarrow \infty} h(t)=0$;

(f) $k$ is such that

$$
0<k<\frac{1}{\lambda|f(G)|}
$$

Then (5.1) has a unique solution $u \in W_{\mathrm{loc}}^{1,1}\left(\mathbb{R}_{+}\right)$and

$$
\lim _{t \rightarrow \infty}(\Phi(g+G u))(t)=\rho-\vartheta, \quad(\Phi(g+G u))^{\prime} \in L^{2}\left(\mathbb{R}_{+}\right) .
$$

In particular, $\lim _{t \rightarrow \infty} e(t)=0$. Moreover, if $\rho-\vartheta$ is an interior point of NVS $\Phi$, then $g+G u$ is bounded.

Remark 5.2. Assumption (a) implies that the step response of $G$ satisfies $G \theta \in\left(L^{2}\left(\mathbb{R}_{+}\right)+\mathbb{R} \theta\right) \cap W_{\text {loc }}^{1,1}\left(\mathbb{R}_{+}\right)$ and $(G \theta)^{\prime} \in L^{2}\left(\mathbb{R}_{+}\right)$.

Proof of Theorem 5.1. Let $u$ be the unique solution of (5.1) (see Th. 7.4 in the Appendix). Let us define an operator $\tilde{\Phi} \in \mathcal{N}(\lambda)$ and a function $w$ by

$$
\tilde{\Phi}(v):=\Phi(v)-(\rho-\vartheta) \theta, \quad \forall v \in C\left(\mathbb{R}_{+}\right) ; \quad w:=g+G u .
$$

Then

and so, by (5.1),

$$
\tilde{\Phi}(w)=\Phi(g+G u)-(\rho-\vartheta) \theta,
$$

$$
u^{\prime}=-k \tilde{\Phi}(w)-k h
$$

Since

we obtain

$$
(G u)(t)=\left(G\left(u(0) \theta+\int_{0}^{\cdot} u^{\prime}(\tau) \mathrm{d} \tau\right)\right)(t)=u(0)(G \theta)(t)+\int_{0}^{t}\left(G u^{\prime}\right)(\tau) \mathrm{d} \tau,
$$

$$
w^{\prime}=g^{\prime}+u(0)(G \theta)^{\prime}+G u^{\prime}=g^{\prime}+u(0)(G \theta)^{\prime}-k G \tilde{\Phi}(w)-k G h .
$$

By Lemma 3.1,

$$
(\tilde{\Phi}(w))^{\prime}=d_{w} w^{\prime}
$$

where $d_{w}: \mathbb{R}_{+} \rightarrow[0, \lambda]$ is a measurable function. Hence,

$$
(\tilde{\Phi}(w))(t)=(\tilde{\Phi}(w))(0)+\int_{0}^{t} d_{w}(\tau) w^{\prime}(\tau) \mathrm{d} \tau .
$$

Substituting this into (5.2) gives

$$
w^{\prime}(t)=r(t)-\int_{0}^{t} G\left(N\left(\cdot, w^{\prime}\right)\right)(\tau) \mathrm{d} \tau, \quad \forall t \geq 0,
$$

where $N(t, \xi):=k d_{w}(t) \xi$ and

$$
r=g^{\prime}+u(0)(G \theta)^{\prime}-k(\tilde{\Phi}(w))(0) G \theta-k G h .
$$

Note that $r \in L^{2}\left(\mathbb{R}_{+}\right)+\mathbb{R} \theta$ due to Remark 5.2 and

$$
0 \leq N(t, \xi) \xi \leq \lambda k \xi^{2}, \quad \forall(t, \xi) \in \mathbb{R}_{+} \times \mathbb{R} .
$$


By assumption (f) on $k$, there exists $0<a<1 /|f(G)|$ such that

$$
0 \leq N(t, \xi) \xi \leq a \xi^{2}, \quad(t, \xi) \in \mathbb{R}_{+} \times \mathbb{R}
$$

Applying Proposition 2.1 to (5.4), we obtain that

$$
N\left(\cdot, w^{\prime}\right)=k d_{w} w^{\prime} \in L^{2}\left(\mathbb{R}_{+}\right)
$$

and the limit

$$
\lim _{t \rightarrow \infty} \int_{0}^{t} N\left(\tau, w^{\prime}(\tau)\right) \mathrm{d} \tau=k \lim _{t \rightarrow \infty} \int_{0}^{t} d_{w}(\tau) w^{\prime}(\tau) \mathrm{d} \tau
$$

exists and is finite. Combining (5.3) and (5.6) we conclude that

$$
\lim _{t \rightarrow \infty}(\Phi(g+G u))(t)=\lim _{t \rightarrow \infty}(\tilde{\Phi}(w))(t)+\rho-\vartheta=(\tilde{\Phi}(w))(0)+\rho-\vartheta+\lim _{t \rightarrow \infty} \int_{0}^{t} d_{w}(\tau) w^{\prime}(\tau) \mathrm{d} \tau
$$

is finite. Furthermore, by (5.5)

$$
(\Phi(g+G u))^{\prime}=(\tilde{\Phi}(w))^{\prime}=d_{w} w^{\prime} \in L^{2}\left(\mathbb{R}_{+}\right) .
$$

It remains to prove that

$$
\lim _{t \rightarrow \infty}(\Phi(g+G u))(t)=\rho-\vartheta
$$

which is equivalent to $\lim _{t \rightarrow \infty}(\tilde{\Phi}(w))(t)=0$. Seeking a contradiction, suppose that $\lim _{t \rightarrow \infty}(\tilde{\Phi}(w))(t)>0($ the case $\lim _{t \rightarrow \infty}(\tilde{\Phi}(w))(t)<0$ can be treated similarly). Note that

$$
G(\tilde{\Phi}(w))=\tilde{f}+\mathbf{G}(0)(\tilde{\Phi}(w))
$$

where $\tilde{f}=(\tilde{\Phi}(w))(0)(G \theta-\mathbf{G}(0))+H\left((\tilde{\Phi}(w))^{\prime}\right) \in L^{2}\left(\mathbb{R}_{+}\right)$with $H$ defined by (4.9). Therefore, by $(5.2), w^{\prime}$ can be written in the form

$$
w^{\prime}=f-k \mathbf{G}(0)(\tilde{\Phi}(w))
$$

where $f \in L^{2}\left(\mathbb{R}_{+}\right)$. Employing Lemma 2.2 with $g=w, f_{1}=1, f_{2}=-k \mathbf{G}(0)(\tilde{\Phi}(w)), f_{3}=-f$, we obtain that the function $w$ is approximately ultimately non-increasing and $w(t) \rightarrow-\infty$ as $t \rightarrow \infty$. This implies, due to (N5), that

$$
\lim _{t \rightarrow \infty}(\tilde{\Phi}(w))(t)=\lim _{t \rightarrow \infty}(\Phi(w))(t)-\rho+\vartheta=\inf \operatorname{NVS} \Phi-\rho+\vartheta
$$

Since $\rho-\vartheta \in \overline{\operatorname{NVS}} \Phi$ by assumption (d),

$$
\rho-\vartheta \geq \inf \operatorname{NVS} \Phi=\lim _{t \rightarrow \infty}(\tilde{\Phi}(w))(t)+\rho-\vartheta
$$

contradicting the supposition that $\lim _{t \rightarrow \infty}(\tilde{\Phi}(w))(t)>0$.

Finally, assume that $\rho-\vartheta$ is an interior point of NVS $\Phi$. Then from (5.7) and (N6) we readily conclude that $g+G u$ is bounded.

\section{Application to WELl-POSED Infinite-Dimensional STATE-SPACE SYSTEMS}

There are a number of equivalent definitions of well-posed systems, see [23-25, 29]. We will be brief in the following and refer the reader to the above references for more details. We wish to consider a well-posed system $\Sigma$ with state-space $X$ (a real Hilbert space with norm denoted by $\|\cdot\|$ ), input space $U=\mathbb{R}$ and output space $Y=\mathbb{R}$, generating operators $(A, B, C)$, input-output operator $G$ and transfer function $\mathbf{G}$. Here $A$ is the 
generator of a strongly continuous semigroup $\mathbf{T}=\left(\mathbf{T}_{t}\right)_{t>0}$ on $X, B \in \mathcal{B}\left(\mathbb{R}, X_{-1}\right)$ and $C \in \mathcal{B}\left(X_{1}, \mathbb{R}\right)$, where $X_{1}$ denotes the domain of $A$ endowed with the norm $\|x\|_{1}:=\|x\|+\|A x\|$ (the graph norm of $A$ ), whilst $X_{-1}$ denotes the completion of $X$ with respect to the norm $\|x\|_{-1}=\left\|(\zeta I-A)^{-1} x\right\|$, where $\zeta \in \operatorname{res}(A)$, the resolvent set of $A$ (different choices of $\zeta$ lead to equivalent norms). Clearly, $X_{1} \subset X \subset X_{-1}$ and the canonical injections are bounded and dense. The semigroup $\mathbf{T}$ restricts to a strongly continuous semigroup on $X_{1}$ and extends to a strongly continuous semigroup on $X_{-1}$ with the exponential growth constant

$$
\omega(\mathbf{T}):=\lim _{t \rightarrow \infty} \frac{\ln \left\|\mathbf{T}_{t}\right\|}{t}
$$

being the same on all three spaces; the generator of the restriction (extension) of $\mathbf{T}$ is a restriction (extension) of $A$; we will use the same symbol $\mathbf{T}$ (respectively, $A$ ) for the original semigroup (respectively, generator) and the associated restrictions and extensions: with this convention, we may write $A \in \mathcal{B}\left(X, X_{-1}\right)$ (considered as a generator on $X_{-1}$, the domain of $A$ is $X$ ). Moreover, the operators $B$ and $C$ are admissible control and observation operators for $\mathbf{T}$, respectively. The transfer function $\mathbf{G}$ of $\Sigma$ is related to the state-space operators $A, B$ and $C$ as follows:

$$
\frac{1}{s-\zeta}(\mathbf{G}(s)-\mathbf{G}(\zeta))=-C(s I-A)^{-1}(\zeta I-A)^{-1} B, \quad \operatorname{Re} s, \operatorname{Re} \zeta>\omega(\mathbf{T}), s \neq \zeta .
$$

The so-called $\Lambda$-extension $C_{\Lambda}$ of $C$ is defined by

$$
C_{\Lambda} z:=\lim _{s \rightarrow \infty, s \in \mathbb{R}} C s(s I-A)^{-1} z
$$

where the domain $D\left(C_{\Lambda}\right)$ of $C_{\Lambda}$ consists of all $z \in X$ for which the above limit exists. For every $z \in X$, $\mathbf{T}_{t} z \in D\left(C_{\Lambda}\right)$ for almost every $t \in \mathbb{R}_{+}$and, if $\omega>\omega(\mathbf{T})$, then the function $t \mapsto \mathrm{e}^{-\omega t} C_{\Lambda} \mathbf{T}_{t} z$ is in $L^{2}\left(\mathbb{R}_{+}\right)$.

For $x^{0} \in X$ and $v \in L_{\text {loc }}^{2}\left(\mathbb{R}_{+}\right)$, let $x$ and $w$ denote the state and output functions of $\Sigma$, respectively, corresponding to the initial condition $x(0)=x^{0} \in X$ and the input function $v$. Then $w=C_{\Lambda} \mathbf{T} x^{0}+G v$, $x(t)=\mathbf{T}_{t} x^{0}+\int_{0}^{t} \mathbf{T}_{t-\tau} B v(s) \mathrm{d} s$ for all $t \in \mathbb{R}_{+}, x(t)+(\zeta I-A)^{-1} B v(t) \in D\left(C_{\Lambda}\right)$ for a.e. $t \in \mathbb{R}_{+}$and

$$
\begin{aligned}
x^{\prime} & =A x+B v, \quad x(0)=x^{0}, \\
w & =C_{\Lambda}\left(x-(\zeta I-A)^{-1} B v\right)+\mathbf{G}(\zeta) v,
\end{aligned}
$$

where $\operatorname{Re} \zeta>\omega(\mathbf{T})$. Of course, (6.2) holds almost everywhere on $\mathbb{R}_{+}$and the differential equation (6.2a) has to be interpreted in $X_{-1}$. In the following, we identify $\Sigma$ and (6.2).

The well-posed system (6.2) is said to be strongly stable if the following four conditions are satisfied:

(i) $G$ is $L^{2}$-stable, i.e., $G \in \mathcal{B}\left(L^{2}\left(\mathbb{R}_{+}\right)\right)$, or, equivalently, $\mathbf{G} \in H^{\infty}\left(\mathbb{C}_{+}\right)$;

(ii) $\mathbf{T}$ is strongly stable, i.e., $\lim _{t \rightarrow \infty} \mathbf{T}_{t} z=0$ for all $z \in X$;

(iii) $B$ is an infinite-time admissible control operator, i.e., there exists $\alpha \geq 0$ such that $\left\|\int_{0}^{\infty} \mathbf{T}_{\tau} B v(\tau) \mathrm{d} \tau\right\| \leq \alpha\|v\|_{L^{2}\left(\mathbb{R}_{+}\right)}$for all $v \in L^{2}\left(\mathbb{R}_{+}\right)$

(iv) $C$ is an infinite-time admissible observation operator, i.e., there exists $\beta \geq 0$ such that $\left(\int_{0}^{\infty}\left\|C \mathbf{T}_{\tau} z\right\|^{2} \mathrm{~d} \tau\right)^{1 / 2} \leq \beta\|z\|$ for all $z \in X_{1}$.

Obviously, exponential stability (i.e., $\omega(\mathbf{T})<0$ ) implies strong stability; the converse is not true.

If the well-posed system (6.2) is regular, i.e. if the following limit

$$
\lim _{s \rightarrow \infty, s \in \mathbb{R}} \mathbf{G}(s)=D
$$


exists and is finite, then $x(t) \in D\left(C_{\Lambda}\right)$ for almost every $t \in \mathbb{R}_{+}$, the output equation (6.2b) simplifies to

$$
y(t)=C_{\Lambda} x(t)+D u(t), \text { a.e. } t \geq 0
$$

and

$$
(G u)(t)=C_{\Lambda} \int_{0}^{t} \mathbf{T}_{t-\tau} B u(\tau) \mathrm{d} \tau+D u(t), \quad \forall u \in L_{\text {loc }}^{2}\left(\mathbb{R}_{+}\right), \text {a.e. } t \in \mathbb{R}_{+} .
$$

Moreover, in the regular case, we have that $(s I-A)^{-1} B \mathbb{R} \subset D\left(C_{\Lambda}\right)$ for all $s \in \operatorname{res}(A)$ and

$$
\mathbf{G}(s)=C_{\Lambda}(s I-A)^{-1} B+D, \quad \operatorname{Re} s>\omega(\mathbf{T}) .
$$

The number $D$ is called the feedthrough of (6.2).

Remark 6.1. It has been known for a considerable time that a large class of retarded and neutral systems (containing all delay systems which are relevant in control engineering) is captured by the theory of well-posed systems (see, for example, $[22,23]$ ). In the last decade, much work has been done to verify well-posedness or regularity for systems described by multidimensional partial differential equations with boundary control and/or boundary observation, see, for example, [4,23] for heat equations, $[10,15,28,30]$ for wave equations, [8] for Schrödinger equations, [9] for the Euler-Bernoulli plate equation and [21,30] for certain structural acoustics models.

Assume that (6.2) is connected in series with a hysteretic input nonlinearity and a static output nonlinearity, the latter of which is subject to output disturbances. Application of integral control to the series interconnection (see Fig. 6) leads to the following feedback law

$$
\begin{aligned}
v & =\Phi(u), \\
y & =\psi(w)+\vartheta+h, \\
u^{\prime} & =\kappa(\rho-y), \quad u(0)=u^{0},
\end{aligned}
$$

where $\Phi$ is a hysteresis operator, $\psi$ is a static nonlinearity, $\kappa$ is a time-varying gain, $\rho, \vartheta \in \mathbb{R}$ and $h$ is the nonconstant part of the output disturbance. A solution of the feedback system given by (6.2) and (6.3) on an interval $[0, \tau)$ is a continuous function $(x, u):[0, \tau) \rightarrow X \times \mathbb{R}$ such that $(x(0), u(0))=\left(x^{0}, u^{0}\right),(x, u) \in$ $W_{\text {loc }}^{1,1}\left([0, \tau), X_{-1} \times \mathbb{R}\right)$ and the equations $(6.2)$ and $(6.3)$ are satisfied almost everywhere on $[0, \tau)$.

The following theorem is a state-space version of Theorem 4.1. Before stating it, we remark that if (6.2) is strongly stable and $0 \in \operatorname{res}(\mathrm{A})$, then $\mathbf{G}$ can be analytically extended from $\mathbb{C}_{+}$to a neighbourhood of 0 . Hence, the evaluation $\mathbf{G}(0)$ of $\mathbf{G}(s)$ at $s=0$ makes sense and (6.1) holds for $\zeta=0$. Consequently, since $\omega(\mathbf{T}) \leq 0$ (by strong stability), we have that

$$
(\mathbf{G}(s)-\mathbf{G}(0)) / s=C(s I-A)^{-1} A^{-1} B, \quad \operatorname{Re} s>0 .
$$

Theorem 6.2. Assume that the following hold:

(a) System (6.2) is strongly stable, $0 \in \operatorname{res}(\mathrm{A})$ and $\mathbf{G}(0)>0$;

(b) $\Phi \in \mathcal{N}\left(\lambda_{1}\right)$;

(c) $\psi: \mathbb{R} \rightarrow \mathbb{R}$ is non-decreasing and globally Lipschitz continuous with Lipschitz constant $\lambda_{2}$;

(d) $\rho-\vartheta \in \mathcal{R}(G, \Phi, \psi)$;

(e) $h$ is such that $h \in L^{1}\left(\mathbb{R}_{+}\right) \cap L^{2}\left(\mathbb{R}_{+}\right)$and the function $t \mapsto \int_{t}^{\infty}|h(\tau)| \mathrm{d} \tau$ is in $L^{2}\left(\mathbb{R}_{+}\right)$;

(f) $\kappa: \mathbb{R}_{+} \rightarrow \mathbb{R}_{+}$is measurable and bounded with

$$
\limsup _{t \rightarrow \infty} \kappa(t)<\frac{1}{\lambda_{1} \lambda_{2}|f(G)|}
$$


where $1 / 0:=\infty$.

Then, for every $\left(x^{0}, u^{0}\right) \in X \times \mathbb{R}$, there exists a unique solution

$$
(x, u) \in C\left(\mathbb{R}_{+}, X \times \mathbb{R}\right) \cap W_{\text {loc }}^{1,1}\left(\mathbb{R}_{+}, X_{-1} \times \mathbb{R}\right)
$$

of the feedback system, given by (6.2) and (6.3), and the following statements hold:

(i) $(\Phi(u))^{\prime} \in L^{2}\left(\mathbb{R}_{+}\right)$and the limit $\Phi^{\infty}:=\lim _{t \rightarrow \infty}(\Phi(u))(t)$ exists and is finite.

(ii) $\lim _{t \rightarrow \infty}\left\|x(t)+A^{-1} B \Phi^{\infty}\right\|=0$.

(iii) The signals $w=C_{\Lambda} \mathbf{T} x^{0}+(G \circ \Phi)(u)$ and $y=\psi(w)+\vartheta+h$ can be decomposed as $w=w_{1}+w_{2}$ and $y=y_{1}+y_{2}$, where $w_{1}, y_{1}$ are continuous and have finite limits

$$
w_{1}^{\infty}:=\lim _{t \rightarrow \infty} w_{1}(t)=\mathbf{G}(0) \Phi^{\infty}, \quad y_{1}^{\infty}:=\lim _{t \rightarrow \infty} y_{1}(t)=\psi\left(\mathbf{G}(0) \Phi^{\infty}\right)+\vartheta,
$$

and $w_{2}, y_{2} \in L^{2}\left(\mathbb{R}_{+}\right)$. If $\lim _{t \rightarrow \infty} h(t)=0$ and, for some $t_{0} \geq 0$,

$$
\mathbf{T}_{t_{0}}\left(A x^{0}+B(\Phi(u))(0)\right) \in X
$$

or

$$
\mathbf{T}_{t_{0}} x^{0} \in X_{1} \quad \text { and } \quad \lim _{t \rightarrow \infty}(G \theta)(t)=\mathbf{G}(0)
$$

we have

$$
\lim _{t \rightarrow \infty} w_{2}(t)=0, \quad \lim _{t \rightarrow \infty} y_{2}(t)=0 .
$$

(iv) If $\kappa \notin L^{1}\left(\mathbb{R}_{+}\right)$, then $y_{1}^{\infty}=\rho$ and the error signal $e=\rho-y$ can be decomposed as $e=e_{1}+e_{2}$, where $e_{1}$ is continuous with $\lim _{t \rightarrow \infty} e_{1}(t)=0$ and $e_{2} \in L^{2}\left(\mathbb{R}_{+}\right)$. If $\lim _{t \rightarrow \infty} h(t)=0$ and, for some $t_{0} \geq 0$, (6.5) or (6.6) holds, then

$$
\lim _{t \rightarrow \infty} e(t)=0
$$

(v) If $\rho-\vartheta$ is an interior point of the set $\mathcal{R}(G, \Phi, \psi)$, then $u$ is bounded.

Proof. It follows from hypothesis (a) that $G$ satisfies assumption (L). Let $\left(x^{0}, u^{0}\right) \in X \times \mathbb{R}$ and set

$$
g:=C_{\Lambda} \mathbf{T} x^{0} .
$$

Strong stability yields that $g \in L^{2}\left(\mathbb{R}_{+}\right)$. By Theorem 4.1, the functional differential equation

$$
u^{\prime}=\kappa(\rho-\vartheta-h-\psi(g+(G \circ \Phi)(u))), \quad u(0)=u^{0}
$$

has a unique solution $u \in W_{\text {loc }}^{1,1}(\mathbb{R})$. Define the continuous function $x: \mathbb{R}_{+} \rightarrow X$ by

$$
x(t)=\mathbf{T}_{t} x^{0}+\int_{0}^{t} \mathbf{T}_{t-\tau} B(\Phi(u))(\tau) \mathrm{d} \tau
$$

Then

$$
g+G \Phi(u)=w=C_{\Lambda}\left(x-(\zeta I-A)^{-1} B \Phi(u)\right)+\mathbf{G}(\zeta) \Phi(u)
$$

and a routine argument using standard properties of well-posed systems shows that $(x, u)$ is the unique solution (defined on $\mathbb{R}_{+}$) of the feedback system given by (6.2) and (6.3). Statements (i) and (v) follow immediately from Theorem 4.1 and statement (ii) is a consequence of part (b) of Lemma 5.2 in [19]. 
To prove statements (iii) and (iv), write $w=w_{1}+w_{2}$ and $y=y_{1}+y_{2}$, where $w_{i}$ and $y_{i}(i=1,2)$ are given by (4.10) and (4.11). Moreover, write $e=e_{1}+e_{2}$, with $e_{1}:=\rho-y_{1}$ and $e_{2}:=-y_{2}$. It follows immediately from Theorem 4.1 that $w_{1}, y_{1}$ and $e_{1}$ are continuous,

$$
w_{1}^{\infty}=\mathbf{G}(0) \Phi^{\infty}, \quad y_{1}^{\infty}=\psi\left(\mathbf{G}(0) \Phi^{\infty}\right)+\vartheta
$$

and $w_{2}, y_{2}, e_{2} \in L^{2}\left(\mathbb{R}_{+}\right)$. Now assume that $\lim _{t \rightarrow \infty} h(t)=0$ and (6.5) or (6.6) hold. To prove that $w_{2}(t)$ and $y_{2}(t)$ converge to 0 as $t \rightarrow \infty$, it is sufficient to show that (4.3) is satisfied (see statement (ii) of Th 4.1). If (6.6) holds, then (4.3) follows trivially. If (6.5) holds, then, setting

$$
f:=g+(\Phi(u))(0)(G \theta-\mathbf{G}(0))
$$

taking Laplace transform and invoking (6.4) and (6.7), we obtain

$$
\begin{aligned}
\hat{f}(s) & \left.=C(s I-A)^{-1} x^{0}+\frac{1}{s}(\mathbf{G}(s)-\mathbf{G}(0)) \Phi(u)\right)(0) \\
& \left.=C(s I-A)^{-1} x^{0}+C(s I-A)^{-1} A^{-1} B \Phi(u)\right)(0) \\
& \left.=C(s I-A)^{-1} A^{-1}\left(A x^{0}+B \Phi(u)\right)(0)\right) .
\end{aligned}
$$

Inverse Laplace transform gives

$$
\left.\left.f(t)=C_{\Lambda} \mathbf{T}_{t} A^{-1}\left(A x^{0}+B \Phi(u)\right)(0)\right)=C A^{-1} \mathbf{T}_{t-t_{0}} \mathbf{T}_{t_{0}}\left(A x^{0}+B \Phi(u)\right)(0)\right), \quad \forall t \geq t_{0} .
$$

Strong stability combined with (6.5) yields that $f(t) \rightarrow 0$ as $t \rightarrow \infty$, showing that (4.3) is satisfied. Finally, assume in addition that $\kappa \notin L^{1}\left(\mathbb{R}_{+}\right)$. Then, by Theorem 4.1, $y_{1}^{\infty}=\rho$ and hence $\lim _{t \rightarrow \infty} e(t)=0$, completing the proof of statements (iii) and (iv).

Finally, we show how Theorem 5.1 can be used in a state-space context. To this end assume that (6.2) is connected in series with a hysteretic output nonlinearity which is subject to output disturbances. Application of integral control to the series interconnection (see Fig. 7) leads to the following feedback law

$$
\begin{aligned}
v & =u, \\
y & =\Phi(w)+\vartheta+h, \\
u^{\prime} & =k(\rho-y), \quad u(0)=u^{0},
\end{aligned}
$$

where $\Phi$ is a hysteresis operator, $k$ is a constant gain, $\rho, \vartheta \in \mathbb{R}$ and $h$ is in $L^{2}\left(\mathbb{R}_{+}\right)$. A solution of the feedback system given by (6.2) and (6.10) on an interval $[0, \tau)$ is a continuous function $(x, u):[0, \tau) \rightarrow X \times \mathbb{R}$ such that $(x(0), u(0))=\left(x^{0}, u^{0}\right)$, the output $w$ of the linear system given by $(6.2 \mathrm{~b})$ is continuous, $(x, u) \in$ $W_{\text {loc }}^{1,1}\left([0, \tau), X_{-1} \times \mathbb{R}\right)$, and the feedback system equations (6.2) and (6.10) are satisfied almost everywhere on $[0, \tau)$.

Theorem 6.3. Assume that the following hold:

(a) System (6.2) is exponentially stable, $\mathbf{G}(0)>0$ and either $B \in \mathcal{B}(\mathbb{R}, X)$ or $C \in \mathcal{B}(X, \mathbb{R})$;

(b) $\Phi \in \mathcal{N}(\lambda)$;

(c) $\rho-\vartheta \in \overline{\operatorname{NVS}} \Phi$;

(d) $h \in L^{2}\left(\mathbb{R}_{+}\right)$and $\lim _{t \rightarrow \infty} h(t)=0$;

(f) $k$ is such that

$$
0<k<\frac{1}{\lambda|f(G)|} .
$$


Then, for every $\left(x^{0}, u^{0}\right) \in X_{1} \times \mathbb{R}$, there exists a unique solution

$$
(x, u) \in C\left(\mathbb{R}_{+}, X \times \mathbb{R}\right) \cap W_{\text {loc }}^{1,1}\left(\mathbb{R}_{+}, X_{-1} \times \mathbb{R}\right)
$$

of the feedback system, given by (6.2) and (6.10), and

$$
\lim _{t \rightarrow \infty}\left(\Phi\left(C \mathbf{T} x^{0}+G u\right)\right)(t)=\rho-\vartheta, \quad\left(\Phi\left(C \mathbf{T} x^{0}+G u\right)\right)^{\prime} \in L^{2}\left(\mathbb{R}_{+}\right) .
$$

In particular, the tracking error

$$
e(t)=\rho-y(t)=\rho-\vartheta-h(t)-\left(\Phi\left(C \mathbf{T} x^{0}+G u\right)\right)(t)
$$

converges to 0 as $t \rightarrow \infty$. Moreover, if $\rho-\vartheta$ is an interior point of $\operatorname{NVS} \Phi$, then $C \mathbf{T} x^{0}+G u$ is bounded.

Proof. It is a trivial consequence of hypothesis (a) that $G$ satisfies assumption (L). Moreover, it follows from [18] and hypothesis (a) that $G$ is a convolution operator with kernel in $L^{2}\left(\mathbb{R}_{+}\right)+\mathbb{R} \delta_{0}$. Let $\left(x^{0}, u^{0}\right) \in X_{1} \times \mathbb{R}$ and set

$$
g:=C \mathbf{T} x^{0} .
$$

A routine argument shows that $g \in W_{\text {loc }}^{1,1}\left(\mathbb{R}_{+}\right)$and $g^{\prime}=C_{\Lambda} \mathbf{T} A x^{0} \in L^{2}\left(\mathbb{R}_{+}\right)$. By Theorem 5.1, the functional differential equation

$$
u^{\prime}=k(\rho-\vartheta-h-\Phi(g+G u)), \quad u(0)=u^{0}
$$

has a unique solution $u \in W_{\text {loc }}^{1,1}(\mathbb{R})$. Define the continuous function $x: \mathbb{R}_{+} \rightarrow X$ by

$$
x(t)=\mathbf{T}_{t} x^{0}+\int_{0}^{t} \mathbf{T}_{t-\tau} B u(\tau) \mathrm{d} \tau .
$$

It is straightforward to show that $(x, u)$ is the unique solution (defined on $\mathbb{R}_{+}$) of the feedback system given by (6.2) and (6.10). The claim now follows from Theorem 5.1.

\section{Appendix}

In this section, we prove (in Th. 7.3) the existence and uniqueness of global solutions of the initial-value problem (4.1) and, for brevity, we simply state (in Th. 7.4) the corresponding result for the initial-value problem (5.1) (the proof of the latter theorem is similar to that of the former). To this end, we first consider the following initial-value problem:

$$
\begin{aligned}
u^{\prime}(t) & =(F(u))(t), & t \geq \alpha, \\
u(t) & =w(t), & t \in[0, \alpha],
\end{aligned}
$$

where $\alpha \geq 0$ and $w \in C([0, \alpha])$ (if $\alpha=0$, then $C([0, \alpha])=\mathbb{R}$ ). We assume that the operator $F: C\left(\mathbb{R}_{+}\right) \rightarrow$ $L_{\text {loc }}^{1}\left(\mathbb{R}_{+}\right)$is causal and satisfies the following hypotheses:

(H1) For all $a \geq 0$ and $v \in C([0, a])$ there exist $\delta>0, \gamma>0$ and a function $f:[0, \gamma] \rightarrow \mathbb{R}_{+}$, with $f(0)=0$, continuous at zero, such that for all $\varepsilon \in(0, \gamma]$

$$
\int_{a}^{a+\varepsilon}\left|\left(F\left(v_{1}\right)\right)(\tau)-\left(F\left(v_{2}\right)\right)(\tau)\right| \mathrm{d} \tau \leq f(\varepsilon) \max _{\tau \in[a, a+\varepsilon]}\left|v_{1}(\tau)-v_{2}(\tau)\right| \quad \forall v_{1}, v_{2} \in \mathcal{C}(v ; \delta, \varepsilon),
$$

where the set $\mathcal{C}(v ; \delta, \varepsilon)$ is defined in (3.1). 
(H2) For all $a>0$ and $v \in C([0, a))$ there exists $c>0$ such that

$$
\int_{0}^{t}|(F(v))(\tau)| \mathrm{d} \tau \leq c\left(1+\max _{\tau \in[0, t]}|v(\tau)|\right), \quad \forall t \in[0, a) .
$$

Let $I$ be an interval of the form $I=[0, T]$ (with $a<T<\infty$ ) or $I=[0, T)$ (with $a<T \leq \infty$ ). A solution of the initial-value problem (7.1) on $I$ is a function $u \in C(I)$ such that $u(t)=w(t)$ for all $t \in[0, \alpha]$, $u$ is locally absolutely continuous on $I \cap[\alpha, \infty)$ and (7.1a) is satisfied almost everywhere on $I \cap[\alpha, \infty)$. Note that $F(u)$ is well-defined when $u$ is a continuous function defined on a finite interval, see Remark 1.1.

Lemma 7.1. For every $\alpha \geq 0$ and every $w \in C([0, \alpha])$, there exists a unique solution $u$ of (7.1) defined on a maximal interval $\left[0, t_{\max }\right)$ with $t_{\max }>\alpha$. Moreover, if $t_{\max }<\infty$, then

$$
\limsup _{t \rightarrow t_{\max }}|u(t)|=\infty .
$$

Remark 7.2. In order to prove the existence and uniqueness of solutions of (4.1) and (5.1) (which is done in Ths. 7.3 and 7.4 below), we apply Lemma 7.1 with $\alpha=0$. However, to prove extended uniqueness (see step 2 in the proof below), it is convenient, even in the case $\alpha=0$, to have existence and uniqueness on a small interval (see step 1 in the proof below) for general $\alpha \geq 0$. For this reason we consider the initial-value problem (7.1) for $\alpha \geq 0$.

Proof of Lemma 7.1. We proceed in several steps.

Step 1. Existence and uniqueness on a small interval.

Let $\delta>0$ and $\gamma>0$ be the parameters whose existence is guaranteed by (H1) with $a=\alpha$ and $v=w$. Let $\Gamma^{\varepsilon}$, parameterized by $\varepsilon \in(0, \gamma]$, denote the operator defined on $C([0, \alpha+\varepsilon])$ by

$$
\left(\Gamma^{\varepsilon}(x)\right)(t):= \begin{cases}w(t), & t \in[0, \alpha] \\ w(\alpha)+\int_{\alpha}^{t}(F(x))(\tau) \mathrm{d} \tau, & t \in(\alpha, \alpha+\varepsilon] .\end{cases}
$$

Endowed with the metric

$$
\left(v_{1}, v_{2}\right) \mapsto \max _{\tau \in[\alpha, \alpha+\varepsilon]}\left|v_{1}(\tau)-v_{2}(\tau)\right|,
$$

$\mathcal{C}(w ; \delta, \varepsilon)$ is a complete metric space. We will prove that for all $\varepsilon \in(0, \gamma]$ sufficiently small, $\Gamma^{\varepsilon}$ is a strict contraction on $\mathrm{C}(w ; \delta, \varepsilon)$. To show that

$$
\Gamma^{\varepsilon}(\mathfrak{C}(w ; \delta, \varepsilon)) \subset \mathcal{C}(w ; \delta, \varepsilon),
$$

for sufficiently small $\varepsilon>0$, define $\tilde{w}$ by

$$
\tilde{w}(t):= \begin{cases}w(t), & 0 \leq t \leq \alpha \\ w(\alpha), & t>\alpha .\end{cases}
$$

If restricted to the interval $[\alpha, \alpha+\varepsilon], \tilde{w}$ belongs to $\mathcal{C}(w ; \delta, \varepsilon)$. We do not distinguish notationally between $\tilde{w}$ and its restriction. 
Let $v \in \mathcal{C}(w ; \delta, \varepsilon)$ and $t \in[\alpha, \alpha+\varepsilon]$. Then, using (H1),

$$
\begin{aligned}
\left|\left(\Gamma^{\varepsilon}(v)\right)(t)-w(\alpha)\right| & \leq \int_{\alpha}^{\alpha+\varepsilon}|(F(v))(\tau)| \mathrm{d} \tau \\
& \leq \int_{\alpha}^{\alpha+\varepsilon}|(F(v))(\tau)-(F(\tilde{w}))(\tau)| \mathrm{d} \tau+\int_{\alpha}^{\alpha+\varepsilon}|(F(\tilde{w}))(\tau)| \mathrm{d} \tau \\
& \leq f(\varepsilon) \max _{\tau \in[\alpha, \alpha+\varepsilon]}|v(\tau)-w(\alpha)|+\tilde{f}(\varepsilon) \\
& \leq f(\varepsilon) \delta+\tilde{f}(\varepsilon),
\end{aligned}
$$

where

$$
\tilde{f}(\varepsilon):=\int_{\alpha}^{\alpha+\varepsilon}|(F(\tilde{w}))(\tau)| \mathrm{d} \tau
$$

Since $f(\varepsilon)$ and $\tilde{f}(\varepsilon)$ converge to 0 as $\varepsilon \downarrow 0$, it follows that, for all sufficiently small $\varepsilon>0$,

$$
\left|\left(\Gamma^{\varepsilon}(v)\right)(t)-w(\alpha)\right| \leq \delta, \quad \forall t \in[\alpha, \alpha+\varepsilon], \forall v \in \mathcal{C}(w ; \delta, \varepsilon) .
$$

Thus $\Gamma^{\varepsilon}(\mathfrak{C}(w ; \delta, \varepsilon)) \subset \mathcal{C}(w ; \delta, \varepsilon)$, provided that $\varepsilon>0$ is sufficiently small. Furthermore, invoking (H1), we obtain that, for all $v_{1}, v_{2} \in \mathcal{C}(w ; \delta, \varepsilon)$ and for all $t \in[\alpha, \alpha+\varepsilon]$,

$$
\left|\left(\Gamma^{\varepsilon}\left(v_{1}\right)\right)(t)-\left(\Gamma^{\varepsilon}\left(v_{2}\right)\right)(t)\right| \leq \int_{\alpha}^{\alpha+\varepsilon}\left|\left(F\left(v_{1}\right)\right)(\tau)-\left(F\left(v_{2}\right)\right)(\tau)\right| \mathrm{d} \tau \leq f(\varepsilon)\left\|v_{1}-v_{2}\right\|_{C([\alpha, \alpha+\varepsilon])} .
$$

Since $f(\varepsilon)<1$ for all sufficiently small $\varepsilon>0$, there exists $\varepsilon^{*} \in(0, \gamma]$ such that $\Gamma^{\varepsilon}$ has a unique fixed point in $\mathcal{C}(w ; \delta, \varepsilon)$ for every $\varepsilon \in\left(0, \varepsilon^{*}\right]$. In particular, there exists a unique solution $u$ of $(7.1)$ in $\mathfrak{C}\left(w ; \delta, \varepsilon^{*}\right)$. However, at this point we cannot exclude the situation that there may exist other solutions on the interval $\left[0, \alpha+\varepsilon^{*}\right]$ which do not belong to this space. To establish uniqueness on a sufficiently small interval, we define

$$
S:=\left\{t \in\left[0, \varepsilon^{*}\right]:|u(\alpha+t)-w(\alpha)|=\delta\right\}, \quad \varepsilon^{* *}:= \begin{cases}\inf S, & S \neq \emptyset \\ \varepsilon^{*}, & S=\emptyset .\end{cases}
$$

It is clear that $\varepsilon^{* *} \in\left(0, \varepsilon^{*}\right]$ and that $u$ restricted to the interval $\left[0, \alpha+\varepsilon^{* *}\right]$ is the unique solution of (7.1) on this interval.

Step 2. Extended uniqueness.

Let $u_{1} \in C\left(\left[0, \alpha_{1}\right)\right)$ and $u_{2} \in C\left(\left[0, \alpha_{2}\right)\right)$ be solutions, where $\alpha_{1}, \alpha_{2}>\alpha$. Set $\beta:=\min \left\{\alpha_{1}, \alpha_{2}\right\}$. We claim that $u_{1}=u_{2}$ on $[0, \beta)$. Seeking a contradiction, suppose that the claim is false. Then

$$
\alpha^{*}:=\inf \left\{t \in[0, \beta): u_{1}(t) \neq u_{2}(t)\right\}<\beta .
$$

By the uniqueness property at the end of Step 1, we have that $\alpha^{*}>\alpha$. Define $w^{*}(t):=u_{1}(t)=u_{2}(t)$ for all $t \in\left[0, \alpha^{*}\right]$. Again by Step 1 (applied in the case $\alpha=\alpha^{*}$ and $w=w^{*}$ ), there exists $0<\varepsilon<\beta-\alpha^{*}$ and a unique solution $u \in C\left(\left[0, \alpha^{*}+\varepsilon\right]\right)$. It follows that $u_{1}(t)=u_{2}(t)=u(t)$ on $\left[0, \alpha^{*}+\varepsilon\right]$, yielding a contradiction to the definition of $\alpha^{*}$.

Step 3. Existence of a maximal solution.

Let $T$ be a set of all $\tau>\alpha$ such that there exists a solution $u^{\tau}$ of $(7.1)$ on the interval $[0, \tau)$. By step 1 , $T \neq \emptyset$. Let $t_{\max }:=\sup T$ and define a function $u:\left[0, t_{\max }\right) \rightarrow \mathbb{R}$ by setting

$$
u(t)=u^{\tau}(t), \quad \text { for } t \in[0, \tau), \text { where } \tau \in T .
$$


By Step 2 the function $u$ is well-defined, i.e., the definition of $u(t)$ for a particular value $t \in\left[0, t_{\max }\right)$ does not depend on the choice of $\tau \in T \cap(t, \infty)$. Moreover, it is clear that $u$ is a maximal solution of (4.1). Uniqueness of this maximal solution follows from Step 2.

Step 4. Unboundedness of $u$ if $t_{\max }<\infty$.

Assume that $t_{\max }<\infty$. Seeking a contradiction, suppose that $u$ is bounded. Integrating (7.1a) gives

$$
u(t)=u(\alpha)+\int_{\alpha}^{t}(F(u))(\tau) \mathrm{d} \tau, \quad \forall t \in\left[\alpha, t_{\max }\right)
$$

By (H2) (with $a=t_{\max }$ and $v=u$ ) there exists $c>0$ such that

$$
\int_{0}^{t}|(F(u))(\tau)| \mathrm{d} \tau \leq c\left(1+\sup _{t \in\left[0, t_{\max }\right]}|u(t)|\right)<\infty, \quad \forall t \in\left[0, t_{\max }\right),
$$

showing that $F(u) \in L^{1}\left(\left[0, t_{\max }\right]\right)$. Combining this with $(7.2)$, we conclude that the $\operatorname{limit}_{\lim } \rightarrow t_{\max } u(t)=$ : $u\left(t_{\max }\right)$ exists and is finite. Using Step $1, u$ can be extended from the interval $\left[\alpha, t_{\text {max }}\right]$ to the right, contradicting the definition of $t_{\max }$.

Theorem 7.3. Under the assumptions of Theorem 4.1, there exists a unique maximal solution u of (4.1) defined on $\mathbb{R}_{+}$(no finite-escape time).

Proof. Define the causal operator $F: C\left(\mathbb{R}_{+}\right) \rightarrow L_{\text {loc }}^{1}\left(\mathbb{R}_{+}\right)$representing the right-hand side of (4.1) by

$$
F(v):=\kappa(\rho-\vartheta-h-\psi(g+(G \circ \Phi)(v))) .
$$

We claim that $F$ satisfies (H1) and (H2). To this end, let $a \geq 0$ and $v \in C([0, a])$. Since $\Phi \in \mathcal{N}\left(\lambda_{1}\right)$, it follows via Remark 3.3 that there exist $\gamma>0$ and $\delta>0$ such that, for all $\varepsilon \in(0, \gamma]$,

$$
\max _{\tau \in[a, a+\varepsilon]}\left|\left(\Phi\left(v_{1}\right)\right)(\tau)-\left(\Phi\left(v_{2}\right)\right)(\tau)\right| \leq \lambda_{1} \max _{\tau \in[a, a+\varepsilon]}\left|v_{1}(\tau)-v_{2}(\tau)\right|, \quad \forall v_{1}, v_{2} \in \mathcal{C}(v ; \delta, \varepsilon) .
$$

Invoking the global Lipschitz property of $\psi$ (with Lipschitz constant $\lambda_{2}$ ), the Hölder inequality and (7.3), yields that, for all $v_{1}, v_{2} \in \mathcal{C}(v ; \delta, \varepsilon)$,

$$
\begin{aligned}
\int_{a}^{a+\varepsilon}\left|\left(F\left(v_{1}\right)\right)(\tau)-\left(F\left(v_{2}\right)\right)(\tau)\right| \mathrm{d} \tau & \leq \lambda_{2}\|\kappa\|_{L^{\infty}\left(\mathbb{R}_{+}\right)} \int_{a}^{a+\varepsilon}\left|\left(G\left(\Phi\left(v_{1}\right)-\Phi\left(v_{2}\right)\right)\right)(\tau)\right| \mathrm{d} \tau \\
& \leq \sqrt{\varepsilon} \lambda_{2}\|\kappa\|_{L^{\infty}\left(\mathbb{R}_{+}\right)}\|G\|\left\|\Phi\left(v_{1}\right)-\Phi\left(v_{2}\right)\right\|_{L^{2}([a, a+\varepsilon])} \\
& \leq \varepsilon \lambda_{2}\|\kappa\|_{L^{\infty}\left(\mathbb{R}_{+}\right)}\|G\|\left\|\Phi\left(v_{1}\right)-\Phi\left(v_{2}\right)\right\|_{C([a, a+\varepsilon])} \\
& \leq \varepsilon \lambda_{1} \lambda_{2}\|\kappa\|_{L^{\infty}\left(\mathbb{R}_{+}\right)}\|G\|\left\|v_{1}-v_{2}\right\|_{C([a, a+\varepsilon])} .
\end{aligned}
$$

Consequently, (H1) holds with

$$
f(\varepsilon):=\varepsilon \lambda_{1} \lambda_{2}\|\kappa\|_{L^{\infty}\left(\mathbb{R}_{+}\right)}\|G\| .
$$

To establish (H2), we take $a>0$ and $v \in C([0, a))$. Then for $t \in[0, a)$ we have

$$
\begin{aligned}
\int_{0}^{t}|(F(v))(\tau)| \mathrm{d} \tau \leq & \|\kappa\|_{L^{\infty}\left(\mathbb{R}_{+}\right)} \int_{0}^{t}|\rho-\vartheta-h(\tau)-\psi(0)| \mathrm{d} \tau+\lambda_{2}\|\kappa\|_{L^{\infty}\left(\mathbb{R}_{+}\right)} \int_{0}^{t}|g(\tau)| \mathrm{d} \tau \\
& +\lambda_{2}\|\kappa\|_{L^{\infty}\left(\mathbb{R}_{+}\right)} \int_{0}^{t}|(G(\Phi(v)))(\tau)| \mathrm{d} \tau
\end{aligned}
$$


Since, by assumption, $g, h \in L^{2}\left(\mathbb{R}_{+}\right)$, it follows that the first two integrands on the right-hand-side of (7.4) are in $L_{\text {loc }}^{1}\left(\mathbb{R}_{+}\right)$. Estimating the last term in (7.4), via the Hölder inequality and (N4), yields

$$
\begin{gathered}
\int_{0}^{t}|(G(\Phi(v)))(\tau)| \mathrm{d} \tau \leq \sqrt{t}\left(\int_{0}^{t}|(G(\Phi(v)))(\tau)|^{2} \mathrm{~d} \tau\right)^{1 / 2} \leq \sqrt{t}\|G\|\left(\int_{0}^{t}|(\Phi(v))(\tau)|^{2} \mathrm{~d} \tau\right)^{1 / 2} \\
\leq t\|G\| \max _{\tau \in[0, t]}|(\Phi(v))(\tau)| \leq t\|G\| \beta\left(1+\max _{\tau \in[0, t]}|v(\tau)|\right), \quad \forall t \in[0, a) .
\end{gathered}
$$

Combining this estimate with (7.4), we see that (H2) holds with

$$
c:=\|\kappa\|_{L^{\infty}\left(\mathbb{R}_{+}\right)}\left(\int_{0}^{a}|\rho-\vartheta-h(\tau)-\psi(0)| \mathrm{d} \tau+\lambda_{2} \int_{0}^{a}|g(\tau)| \mathrm{d} \tau+\lambda_{2} a\|G\| \beta\right) .
$$

From Lemma 7.1 we conclude that there exists a unique solution $u$ of (4.1) defined on a maximal interval $\left[0, t_{\max }\right)$.

It remains to prove that $u$ has no finite-escape time, i.e., $t_{\max }=\infty$. To this end we take $\sigma \in\left[0, t_{\max }\right)$. Integrating (4.1) from 0 to $\sigma$, we obtain

$$
u(\sigma)=u(0)+\int_{0}^{\sigma} \kappa(\tau)(\rho-\vartheta-h(\tau)-\psi[g(\tau)+(G(\Phi(u)))(\tau)]) \mathrm{d} \tau,
$$

which implies

$$
\begin{aligned}
\max _{\sigma \in[0, t]}|u(\sigma)| \leq & |u(0)|+\|\kappa\|_{L^{\infty}\left(\mathbb{R}_{+}\right)} \int_{0}^{t}|\rho-\vartheta-h(\tau)-\psi(0)| \mathrm{d} \tau \\
& +\|\kappa\|_{L^{\infty}\left(\mathbb{R}_{+}\right)} \lambda_{2} \int_{0}^{t}|g(\tau)| \mathrm{d} \tau+\|\kappa\|_{L^{\infty}\left(\mathbb{R}_{+}\right)} \lambda_{2} \int_{0}^{t}|(G(\Phi(u)))(\tau)| \mathrm{d} \tau, \quad \forall t \in\left[0, t_{\max }\right) .
\end{aligned}
$$

Seeking a contradiction, suppose that $t_{\max }<\infty$. Estimating the last integral in (7.5) and using (N4) (with $\left.a=t_{\max }\right)$ gives

$$
\begin{aligned}
& \int_{0}^{t}|(G(\Phi(u)))(\tau)| \mathrm{d} \tau \leq \sqrt{t}\|G\|\left(\int_{0}^{t} \max _{\sigma \in[0, \tau]}|(\Phi(u))(\sigma)|^{2} \mathrm{~d} \tau\right)^{1 / 2} \\
& \quad \leq \sqrt{t_{\max }}\|G\| \beta\left(\int_{0}^{t}\left(1+\max _{\sigma \in[0, \tau]}|u(\sigma)|\right)^{2} \mathrm{~d} \tau\right)^{1 / 2}, \quad \forall t \in\left[0, t_{\max }\right) .
\end{aligned}
$$

Define $U:\left[0, t_{\max }\right) \rightarrow \mathbb{R}_{+}$by $U(t):=\max _{\sigma \in[0, t]}|u(\sigma)|$. From (7.5) and (7.6) we infer that there exist positive constants $\beta_{1}$ and $\beta_{2}$ such that

$$
U(t) \leq \beta_{1}+\beta_{2}\left(\int_{0}^{t}\left(1+U^{2}(\tau)\right) \mathrm{d} \tau\right)^{1 / 2}, \quad \forall t \in\left[0, t_{\max }\right) .
$$

Consequently,

$$
U^{2}(t) \leq 2 \beta_{1}^{2}+2 \beta_{2}^{2} t_{\max }+2 \beta_{2}^{2} \int_{0}^{t} U^{2}(\tau) \mathrm{d} \tau, \quad \forall t \in\left[0, t_{\max }\right) .
$$

An application of the Gronwall's lemma shows that $U^{2}(t) \leq\left(2 \beta_{1}^{2}+2 \beta_{2}^{2} t_{\max }\right) \exp \left(2 \beta_{2}^{2} t_{\max }\right)$ for all $t \in\left[0, t_{\max }\right)$. Therefore $u$ is bounded on $\left[0, t_{\max }\right)$, contradicting, via Lemma 7.1 , the supposition $t_{\max }<\infty$.

We proceed to state the corresponding existence and uniqueness result for the initial-value problem (5.1): the proof, which is based on Lemma 7.1, is similar to that of Theorem 7.3 and is omitted here. 
Theorem 7.4. Under the assumptions of Theorem 5.1, there exists a unique maximal solution u of (5.1) defined on $\mathbb{R}_{+}$(no finite-escape time).

\section{REFERENCES}

[1] H.T. Banks, R.C. Smith and Y. Wang, Smart Material Structures: Modeling, Estimation, Control. Masson, Paris (1996).

[2] M. Brokate, Hysteresis operators, in Phase Transitions and Hysteresis, A. Visintin Ed., Springer, Berlin (1994) 1-38.

[3] M. Brokate and J. Sprekels, Hysteresis and Phase Transitions, Springer, New York (1996).

[4] C.I. Byrnes, D.S. Gilliam, V.I. Shubov and G. Weiss, Regular linear systems governed by a boundary controlled heat equation. J. Dynam. Control Syst. 8 (2002) 341-370.

[5] R.F. Curtain, H. Logemann and O. Staffans, Stability results of Popov-type for infinite-dimensional systems with applications to integral control. Proc. London Math. Soc. 86 (2003) 779-816.

[6] T. Fliegner, H. Logemann and E.P. Ryan, Low-gain integral control of well-posed infinite-dimensional linear systems with input and output nonlinearities. J. Math. Anal. Appl. 261 (2001) 307-336.

[7] R.B. Gorbet, K.A. Morris and W.L. Wang, Passivity-based stability and control of hysteresis in smart actuators. IEEE Trans. Control Systems Technology 9 (2001) 5-16.

[8] B.Z. Guo and Z.C. Shao, Regularity of a Schrödinger equation with Dirichlet control and colocated observation, Syst. Control Lett. 54 (2005) 1135-1142.

[9] B.Z. Guo and Z.C. Shao, Regularity of an Euler-Bernoulli plate with Neumann control and colocated observation, J. Dynam. Control Syst. 12 (2006) 405-418.

[10] B.Z. Guo and X. Zhang, The regularity of the wave equation with partial Dirichlet control and colocated observation, SIAM J. Control Optim. 44 (2005) 1598-1613.

[11] F. Ikhouane and J. Rodellar, A linear controller for hysteretic systems. IEEE Trans. Auto. Control 51 (2006) $340-344$.

[12] F. Ikhouane, V. Mañosa and J. Rodellar, Adaptive control of a hysteretic structural system. Automatica 41 (2005) 225-231.

[13] U. Jönson, Stability of uncertain systems with hysteresis nonlinearities. Int. J. Robust Nonlinear Control 8 (1998) $279-293$.

[14] M.A. Krasnosel'skii and A.V. Pokrovskii, Systems with Hysteresis. Springer, Berlin (1989).

[15] I. Lasiecka and R. Triggiani, The operator $B^{*} L$ for the wave equation with Dirichlet control. Abstract Appl. Anal. 2004 (2004) 625-634.

[16] H. Logemann and A.D. Mawby, Low-gain integral control of infinite-dimensional regular linear systems subject to input hysteresis, F. Colonius et al. Eds., Birkhäuser, Boston, Advances in Mathematical Systems Theory (2001) 255-293.

[17] H. Logemann and A. Mawby, Discrete-time and sampled-data low-gain integral control of infinite-dimensional linear systems in the presence of input hysteresis. SIAM J. Control Optim. 41 (2002) 113-140.

[18] H. Logemann and E.P. Ryan, Time-varying and adaptive integral control of infinite-dimensional regular systems with input nonlinearities, SIAM J. Control Optim. 38 (2000) 1120-1144.

[19] H. Logemann and E.P. Ryan, Systems with hysteresis in the feedback loop: existence, regularity and asymptotic behaviour of solutions. ESAIM: COCV 9 (2003) 169-196.

[20] H. Logemann, E.P. Ryan and S. Townley, Integral control of linear systems with actuator nonlinearities: lower bounds for the maximal regulating gain. IEEE Trans. Auto. Control 44 (1999) 1315-1319.

[21] R. Rebarber and G. Weiss, Internal model based tracking and disturbance rejection for stable well-posed systems, Automatica 39 (2003) 1555-1569.

[22] D. Salamon, Control and Observation of Neutral Systems. Pitman, London (1984).

[23] D. Salamon, Infinite-dimensional linear systems with unbounded control and observation: a functional analytic approach. Trans. Amer. Math. Soc. 300 (1987) 383-431.

[24] O.J. Staffans, Well-Posed Linear Systems. Cambridge University Press, Cambridge (2005).

[25] O.J. Staffans and G. Weiss, Transfer functions of regular linear systems, part II: The system operator and the Lax-Phillips semigroup. Trans. Amer. Math. Soc. 354 (2002) 3229-3262.

[26] X. Tan and J.S. Baras, Modeling and control of hysteresis in magnetostrictive actuators. Automatica 40 (2004) 1469-1480.

[27] G. Tao and P.V. Kokotović, Adaptive Control of Systems with Actuator and Sensor Nonlinearities. John Wiley, (1996)

[28] M. Tucsnak and G. Weiss, How to get a conservative well-posed system out of thin air, part II: Controllability and stability. SIAM J. Control Optim. 42 (2003) 907-935.

[29] G. Weiss, Transfer functions of regular linear systems, part I: Characterization of regularity. Trans. Amer. Math. Soc. 342 (1994) 827-854.

[30] G. Weiss and R. Rebarber, Optimizability and estimatability for infinite-dimensional linear systems. SIAM J. Control Optim. 39 (2000) 1204-1232. 